\title{
Lagrangian Mechanics and Reduction on Fibered Manifolds
}

\author{
Songhao LI, Ari STERN and Xiang TANG \\ Department of Mathematics, Washington University in St. Louis, \\ One Brookings Drive, St. Louis MO 63130-4899, USA \\ E-mail: lisonghao@gmail.com,stern@wustl.edu,xtang@wustl.edu
}

Received October 05, 2016, in final form March 13, 2017; Published online March 22, 2017

https://doi.org/10.3842/SIGMA.2017.019

\begin{abstract}
This paper develops a generalized formulation of Lagrangian mechanics on fibered manifolds, together with a reduction theory for symmetries corresponding to Lie groupoid actions. As special cases, this theory includes not only Lagrangian reduction (including reduction by stages) for Lie group actions, but also classical Routh reduction, which we show is naturally posed in this fibered setting. Along the way, we also develop some new results for Lagrangian mechanics on Lie algebroids, most notably a new, coordinate-free formulation of the equations of motion. Finally, we extend the foregoing to include fibered and Lie algebroid generalizations of the Hamilton-Pontryagin principle of Yoshimura and Marsden, along with the associated reduction theory.
\end{abstract}

Key words: Lagrangian mechanics; reduction; fibered manifolds; Lie algebroids; Lie groupoids

2010 Mathematics Subject Classification: 70G45; 53D17; 37J15

\section{Introduction}

The starting point for classical Lagrangian mechanics is a function $L: T Q \rightarrow \mathbb{R}$, called the Lagrangian, where $T Q$ is the tangent bundle of a smooth configuration manifold $Q$.

Yet, tangent bundles are hardly the only spaces on which one may wish to study Lagrangian mechanics. When $L$ is invariant with respect to certain symmetries, it is useful to perform Lagrangian reduction: quotienting out the symmetries and thereby passing to a smaller space than $T Q$. For example, if a Lie group $G$ acts freely and properly on $Q$, then $Q \rightarrow Q / G$ is a principal fiber bundle; if $L$ is invariant with respect to the $G$-action, then one can define a reduced Lagrangian on the quotient $T Q / G$ (cf. Marsden and Scheurle [21], Cendra et al. [3]). In particular, when $Q=G$, the reduced Lagrangian is defined on $T G / G \cong \mathfrak{g}$, the Lie algebra of $G$, and the reduction procedure is called Euler-Poincaré reduction (cf. Marsden and Ratiu [18, Chapter 13]).

Unlike $T Q$, the reduced spaces $T Q / G$ and $\mathfrak{g}$ are not tangent bundles - but all three are examples of Lie algebroids. Beginning with a seminal paper of Weinstein [30], and with particularly important follow-up work by Martínez [23, 24, 25], this has driven the development of a more general theory of Lagrangian mechanics on Lie algebroids. In this more general framework, reduction is associated with Lie algebroid morphisms, of which the quotient map $T Q \rightarrow T Q / G$ is a particular example. Since Lie algebroids form a category, the composition of two morphisms is again a morphism. As an important consequence, it is almost trivial to perform so-called reduction by stages - applying a sequence of morphisms one at a time rather than all at once whereas, without this framework, reduction by stages is considerably more difficult (Cendra et al. [3], Marsden et al. [17]).

This paper is a contribution to the Special Issue "Gone Fishing". The full collection is available at http://www.emis.de/journals/SIGMA/gone-fishing2016.html 
In this paper, we generalize the foregoing theory in a new direction, based on the observation that reduction from $T Q$ to $T Q / G$ is a special case of a much more general construction, involving Lie groupoid (rather than group) actions on fibered manifolds (rather than ordinary manifolds). This includes not only Lagrangian reduction, but also the related theory of Routh reduction, which we show is naturally posed in the language of fibered manifolds. In the special case of a manifold trivially fibered over a single point, i.e., an ordinary manifold, this reduces to the previously-studied cases. Along the way, we also develop some new results on Lagrangian mechanics on Lie algebroids - most notably a new, coordinate-free formulation of the equations of motion, incorporating the notion of a Lie algebroid connection due to Crainic and Fernandes [6] and extend this theory to the Hamilton-Pontryagin principle of Yoshimura and Marsden [33].

The paper is organized as follows:

- In Section 2, we begin by briefly reviewing the classical formulation of Lagrangian mechanics on manifolds. We then define fibered manifolds, together with appropriate spaces of vertical tangent vectors and paths, and show how Lagrangian mechanics may be generalized to this setting. As an application, we show that Routh reduction is naturally posed in the language of fibered manifolds, where the classical Routhian is understood as a Lagrangian on an appropriate vertical bundle.

- In Section 3, we discuss Lagrangian mechanics on Lie algebroids. We call the associated equations of motion the Euler-Lagrange-Poincaré equations, since they simultaneously generalize the Euler-Lagrange equations on $T Q$, Euler-Poincaré equations on $\mathfrak{g}$, and Lagrange-Poincaré equations on $T Q / G$. We derive a new, coordinate-free formulation of these equations, which we show agrees with the local-coordinates expression previously obtained by Martínez [23]. Finally, we show that, since the vertical bundle of a fibered manifold is a Lie algebroid, the theory of Section 2 can be interpreted in this light.

- In Section 4, we employ the Lie algebroid toolkit of Section 3 to study Lagrangian reduction on fibered manifolds by Lie groupoid actions, which we call Euler-Lagrange-Poincaré reduction. In the special case where a Lie groupoid acts on itself by multiplication, we recover the theory of Lagrangian mechanics on its associated Lie algebroid.

- Finally, in Section 5, we generalize the Hamilton-Pontryagin variational principle of Yoshimura and Marsden [33], together with the associated reduction theory [34], to fibered manifolds with Lie groupoid symmetries.

\section{Lagrangian mechanics on fibered manifolds}

\subsection{Brief review of Lagrangian mechanics}

Let $Q$ be a smooth configuration manifold and $L: T Q \rightarrow \mathbb{R}$ be a smooth function, called the Lagrangian, on its tangent bundle. There are three ways in which one can use $L$ to induce dynamics on $Q$.

The first, which we call the symplectic approach, begins by introducing the Legendre transform (or fiber derivative) of $L$, which is the bundle map $\mathbb{F} L: T Q \rightarrow T^{*} Q$ defined fiberwise by

$$
\left\langle\mathbb{F}_{q} L(v), w\right\rangle=\left.\frac{\mathrm{d}}{\mathrm{d} t} L(v+t w)\right|_{t=0}, \quad v, w \in T_{q} Q .
$$

This is used to pull back the canonical symplectic form $\omega \in \Omega^{2}\left(T^{*} Q\right)$ to the Lagrangian 2-form $\omega_{L}=(\mathbb{F} L)^{*} \omega \in \Omega^{2}(T Q)$. The Lagrangian is said to be regular if $\mathbb{F} L$ is a local bundle isomorphism; in this case, $\omega_{L}$ is nondegenerate, so $\left(T Q, \omega_{L}\right)$ is a symplectic manifold. The energy function $E_{L}: T Q \rightarrow \mathbb{R}$ associated to $L$ is

$$
E_{L}(v)=\langle\mathbb{F} L(v), v\rangle-L(v),
$$


and the Lagrangian vector field $X_{L} \in \mathfrak{X}(T Q)$ is the vector field satisfying

$$
i_{X_{L}} \omega_{L}=\mathrm{d} E_{L},
$$

where $i_{X_{L}} \omega_{L}=\omega_{L}\left(X_{L}, \cdot\right)$ is the interior product of $X_{L}$ with $\omega_{L}$. That is, $X_{L}$ is the Hamiltonian vector field of $E_{L}$ on the symplectic manifold $\left(T Q, \omega_{L}\right)$. Finally, a $C^{2}$ path $q: I \rightarrow Q$ is called a base integral curve of $X_{L}$ if its tangent prolongation $(q, \dot{q}): I \rightarrow T Q$ is an integral curve of $X_{L}$. (Here and henceforth, $I$ denotes the closed unit interval $[0,1]$, but there is no loss of generality over any other closed interval $[a, b]$.)

The second, which we call the variational approach, begins with the action functional $S: \mathcal{P}(Q) \rightarrow \mathbb{R}$, defined by the integral

$$
S(q)=\int_{0}^{1} L(q(t), \dot{q}(t)) \mathrm{d} t
$$

where $\mathcal{P}(Q)$ denotes the Banach manifold of $C^{2}$ paths $q: I \rightarrow Q$. A path $q \in \mathcal{P}(Q)$ satisfies Hamilton's variational principle if it is a critical point of $S$ restricted to paths with fixed endpoints $q(0)$ and $q(1)$, i.e., if $\mathrm{d} S(\delta q)=0$ for all variations $\delta q \in T_{q} \mathcal{P}(Q)$ with $\delta q(0)=0$ and $\delta q(1)=0$.

The third and final approach considers the system of differential equations that a solution to Hamilton's variational principle must satisfy. In local coordinates, assuming $\delta q(0)=0$ and $\delta q(1)=0$

$$
\mathrm{d} S(\delta q)=\int_{0}^{1}\left(\frac{\partial L}{\partial q^{i}}(q, \dot{q}) \delta q^{i}+\frac{\partial L}{\partial \dot{q}^{i}}(q, \dot{q}) \delta \dot{q}^{i}\right) \mathrm{d} t=\int_{0}^{1}\left(\frac{\partial L}{\partial q^{i}}(q, \dot{q})-\frac{\mathrm{d}}{\mathrm{d} t} \frac{\partial L}{\partial \dot{q}^{i}}(q, \dot{q})\right) \delta q^{i} \mathrm{~d} t .
$$

(Here, we use the Einstein index convention, where there is an implicit sum on repeated indices.) Hence, this vanishes for all $\delta q$ if and only if $q$ satisfies the system of ordinary differential equations

$$
\frac{\partial L}{\partial q^{i}}(q, \dot{q})-\frac{\mathrm{d}}{\mathrm{d} t} \frac{\partial L}{\partial \dot{q}^{i}}(q, \dot{q})=0,
$$

which are called the Euler-Lagrange equations.

The equivalence of these three approaches for regular Lagrangians - and of the latter two for arbitrary Lagrangians - is a standard result in geometric mechanics. We state it now as a theorem for later reference.

Theorem 2.1. If $L: T Q \rightarrow \mathbb{R}$ is a regular Lagrangian and $q \in \mathcal{P}(Q)$, then the following are equivalent:

(i) $q$ is a base integral curve of the Lagrangian vector field $X_{L} \in \mathfrak{X}(T Q)$.

(ii) q satisfies Hamilton's variational principle.

(iii) q satisfies the Euler-Lagrange equations.

If regularity is dropped, then $($ ii $) \Leftrightarrow($ iii $)$ still holds.

Proof. See, e.g., Marsden and Ratiu [18, Theorem 8.1.3].

\section{$2.2 \quad$ Fibered manifolds}

We begin by giving the definition of a fibered manifold, along with its vertical and covertical bundles. These bundles generalize the tangent and cotangent bundles of an ordinary manifold, and they will play analogous roles in fibered Lagrangian mechanics. 
Definition 2.2. A fibered manifold $Q \rightarrow M$ consists of a pair of smooth manifolds $Q, M$, together with a surjective submersion $\mu: Q \rightarrow M$.

Definition 2.3. The vertical bundle of $Q \rightarrow M$ is $V Q=\operatorname{ker} \mu_{*}$, where $\mu_{*}: T Q \rightarrow T M$ is the pushforward (or tangent map) of $\mu$. The dual of $V Q$ is denoted $V^{*} Q$, which we call the covertical bundle.

Remark 2.4. Since $\mu$ is a submersion, the fiber $Q_{x}=\mu^{-1}(\{x\})$ is a submanifold of $Q$ for each $x \in M$. Therefore,

$$
V_{q} Q=T_{q} Q_{\mu(q)}, \quad V Q=\bigsqcup_{q \in Q} V_{q} Q=\bigsqcup_{x \in M} T Q_{x}
$$

In other words, $V Q$ consists of vectors tangent to the fibers, and hence is an integrable subbundle of $T Q$. Similarly,

$$
V_{q}^{*} Q=T_{q}^{*} Q_{\mu(q)}, \quad V^{*} Q=\bigsqcup_{q \in Q} V_{q}^{*} Q=\bigsqcup_{x \in M} T^{*} Q_{x},
$$

so the covertical bundle consists of covectors to the individual fibers.

Example 2.5. An ordinary smooth manifold $Q$ can be identified with the fibered manifold $Q \rightarrow \bullet$, where $\bullet$ denotes the space with a single point. Because $\mu_{*}$ is trivial, it follows that $V Q=T Q$ and $V^{*} Q=T^{*} Q$.

Definition 2.6. The space of vertical vector fields on $Q$ is $\mathfrak{X}_{V}(Q)=\Gamma(V Q)$. The space of vertical $k$-forms on $Q$ is $\Omega_{V}^{k}(Q)=\Gamma\left(\bigwedge^{k} V^{*} Q\right)$.

Since $V Q$ is integrable, it follows that $\mathfrak{X}_{V}(Q)$ is closed under the Jacobi-Lie bracket $[\cdot, \cdot]$, i.e., $\mathfrak{X}_{V}(Q)$ is a Lie subalgebra of $\mathfrak{X}(Q)$. Therefore, the following vertical exterior derivative operator on $\Omega_{V}^{\bullet}(Q)$ is well-defined.

Definition 2.7. Given $u \in \Omega_{V}^{k}(Q)$, the vertical exterior derivative $\mathrm{d}_{V} u \in \Omega_{V}^{k+1}(Q)$ is given by

$$
\begin{aligned}
\mathrm{d}_{V} u\left(X_{0}, \ldots, X_{k}\right)= & \sum_{i=0}^{k}(-1)^{i} X_{i}\left[u\left(X_{0}, \ldots, \widehat{X}_{i}, \ldots, X_{k}\right)\right] \\
& +\sum_{0 \leq i<j \leq k}(-1)^{i+j}\left(\left[X_{i}, X_{j}\right], X_{0}, \ldots, \widehat{X}_{i}, \ldots, \widehat{X}_{j}, \ldots, X_{k}\right),
\end{aligned}
$$

where $X_{0}, \ldots, X_{k} \in \mathfrak{X}_{V}(Q)$ are arbitrary vertical vector fields, and where a hat over an argument indicates its omission.

Remark 2.8. From the characterization of $V Q$ in Remark 2.4, it follows that $X \in \mathfrak{X}_{V}(Q)$ restricts to an ordinary vector field $X_{x} \in \mathfrak{X}\left(Q_{x}\right)$ on each fiber $Q_{x}$. Likewise, $u \in \Omega_{V}^{k}(Q)$ restricts to an ordinary $k$-form $u_{x} \in \Omega^{k}\left(Q_{x}\right)$ on each fiber $Q_{x}$. Moreover, by the integrability of $V Q$, for any $X, Y \in \mathfrak{X}_{V}(Q)$ and $x \in M$ we have $[X, Y]_{x}=\left[X_{x}, Y_{x}\right] \in \mathfrak{X}\left(Q_{x}\right)$. Hence, the vertical exterior derivative $\mathrm{d}_{V}$ coincides with the ordinary exterior derivative $\mathrm{d}_{x}: \Omega^{k}\left(Q_{x}\right) \rightarrow \Omega^{k+1}\left(Q_{x}\right)$ on the fiber $Q_{x}$.

Note that $V Q$ and $V^{*} Q$ are also, themselves, fibered manifolds over $M$. Specifically, if $\tau: V Q \rightarrow Q$ and $\pi: V^{*} Q \rightarrow Q$ are the bundle projections, then we have surjective submersions $\mu \circ \tau: V Q \rightarrow M$ and $\mu \circ \pi: V^{*} Q \rightarrow M$; the fibers are given by $(V Q)_{x}=T Q_{x}$ and $\left(V^{*} Q\right)_{x}=T^{*} Q_{x}$. Now, just as there is a tautological 1-form and canonical symplectic 2-form on $T^{*} Q$, there are corresponding vertical forms on $V^{*} Q$, constructed as follows. 
Definition 2.9. The tautological vertical 1-form $\theta \in \Omega_{V}^{1}\left(V^{*} Q\right)$ is defined by the condition $\theta(v)=$ $\left\langle p, \pi_{*} v\right\rangle$ for $v \in V_{p} V^{*} Q$. The canonical vertical 2 -form is defined by $\omega=-\mathrm{d}_{V} \theta \in \Omega_{V}^{2}\left(V^{*} Q\right)$.

Remark 2.10. Restricted to any fiber $\left(V^{*} Q\right)_{x}=T^{*} Q_{x}$, it follows from the preceding remarks that $\theta$ and $\omega$ agree with the ordinary tautological 1-form $\theta_{x} \in \Omega^{1}\left(T^{*} Q_{x}\right)$ and canonical symplectic 2-form $\omega_{x} \in \Omega^{2}\left(T^{*} Q_{x}\right)$, respectively, on the cotangent bundle of the fiber. In particular, this implies that $\omega$ is closed (with respect to $\mathrm{d}_{V}$ ) and nondegenerate, since $\omega_{x}$ is closed and nondegenerate for each $x \in M$.

\subsection{Lagrangian mechanics on fibered manifolds}

In this section, we show that the three approaches to Lagrangian mechanics of Section 2.1 may be generalized to fibered manifolds, with a corresponding generalization of Theorem 2.1. Let the Lagrangian be a smooth function $L: V Q \rightarrow \mathbb{R}$.

Definition 2.11. The Legendre transform (or fiber derivative) of $L$ is the bundle map $\mathbb{F} L: V Q \rightarrow$ $V^{*} Q$, defined for each $q \in Q$ by

$$
\left\langle\mathbb{F}_{q} L(v), w\right\rangle=\left.\frac{\mathrm{d}}{\mathrm{d} t} L(v+t w)\right|_{t=0}, \quad v, w \in V_{q} Q .
$$

We say that $L$ is regular if $\mathbb{F} L$ is a local bundle isomorphism.

Remark 2.12. Since $(V Q)_{x}=T Q_{x}$, we can define a fiber-restricted Lagrangian $L_{x}: T Q_{x} \rightarrow \mathbb{R}$, whose ordinary Legendre transform $\mathbb{F} L_{x}: T Q_{x} \rightarrow T^{*} Q_{x}$ coincides with the restriction $\left.\mathbb{F} L\right|_{(V Q)_{x}}$. It is therefore useful to think of $L$ as a smoothly varying family of ordinary Lagrangians $L_{x}$, parametrized by $x \in M$.

Now, $\mathbb{F} L$ maps fibers to fibers (i.e., it is a morphism of fibered manifolds over $M$ ), so its pushforward maps vertical vectors to vertical vectors, and we may write $(\mathbb{F} L)_{*}: V V Q \rightarrow V V^{*} Q$. This also gives a well-defined pullback of vertical forms $(\mathbb{F} L)^{*}: \Omega_{V}^{k}\left(V^{*} Q\right) \rightarrow \Omega_{V}^{k}(V Q)$, which leads to the following vertical versions of the Lagrangian 2-form and Lagrangian vector field.

Definition 2.13. The Lagrangian vertical 2-form is $\omega_{L}=(\mathbb{F} L)^{*} \omega \in \Omega_{V}^{2}(V Q)$. The Lagrangian vertical vector field $X_{L} \in \mathfrak{X}_{V}(V Q)$ is the vertical vector field satisfying

$$
i_{X_{L}} \omega_{L}=\mathrm{d}_{V} E_{L}
$$

where the energy function $E_{L}: V Q \rightarrow \mathbb{R}$ is given by $E_{L}(v)=\langle\mathbb{F} L(v), v\rangle-L(v)$.

Remark 2.14. Restricting to the fiber over $x \in M$, we have

$$
\left(\omega_{L}\right)_{x}=\left(\mathbb{F} L_{x}\right)^{*} \omega_{x}=\omega_{L_{x}} \in \Omega^{2}\left(T Q_{x}\right),
$$

i.e., the ordinary Lagrangian 2 -form for $L_{x}$ on $T Q_{x}$, and moreover

$$
\left(E_{L}\right)_{x}(v)=\left\langle\mathbb{F} L_{x}(v), v\right\rangle-L_{x}(v)=E_{L_{x}}(v), \quad v \in T Q_{x},
$$

so $E_{L}$ restricts to $E_{L_{x}}$. Combining these, it follows that

$$
i_{\left(X_{L}\right)_{x}} \omega_{L_{x}}=\mathrm{d}_{x} E_{L_{x}}
$$

so we conclude that $\left(X_{L}\right)_{x}=X_{L_{x}}$, i.e., $X_{L}$ coincides with the ordinary Lagrangian vector field on each fiber. 
Next, for the variational approach, we begin by defining an appropriate space of vertical paths on which the action functional will be defined, as well as an appropriate space of variations of these paths.

Definition 2.15. The space of $C^{2}$ vertical paths, denoted by $\mathcal{P}_{V}(Q) \subset \mathcal{P}(Q)$, consists of $q \in \mathcal{P}(Q)$ whose tangent prolongation satisfies $(q(t), \dot{q}(t)) \in V Q$ for all $t \in I$. The action functional $S: \mathcal{P}_{V}(Q) \rightarrow \mathbb{R}$ is then

$$
S(q)=\int_{0}^{1} L(q(t), \dot{q}(t)) \mathrm{d} t
$$

which is well-defined for $L: V Q \rightarrow \mathbb{R}$ since $(q(t), \dot{q}(t)) \in V Q$.

Remark 2.16. For $q \in \mathcal{P}_{V}(Q)$, the condition $(q(t), \dot{q}(t)) \in V Q$ implies

$$
\frac{\mathrm{d}}{\mathrm{d} t} \mu(q(t))=\mu_{*}(q(t), \dot{q}(t))=0 .
$$

Hence, $\mu(q(t))$ is constant in $t$, so $q$ lies in a single fiber $Q_{x}$, i.e., $q \in \mathcal{P}\left(Q_{x}\right)$ for some $x \in M$. It follows that $S(q)=S_{x}(q)$, where $S_{x}: \mathcal{P}\left(Q_{x}\right) \rightarrow \mathbb{R}$ is the ordinary action associated to the fiber-restricted Lagrangian $L_{x}$. Moreover, since $\mu(q(t))$ is constant in $t$, there is an associated fibered (Banach) manifold structure $\mathcal{P}_{V}(Q) \rightarrow M$, with $\mathcal{P}_{V}(Q)_{x}=\mathcal{P}\left(Q_{x}\right)$.

Definition 2.17. An element $\delta q \in V_{q} \mathcal{P}_{V}(Q)$ is called a vertical variation of $q \in \mathcal{P}_{V}(Q)$. The path $q$ satisfies Hamilton's variational principle for vertical paths if $q$ is a critical point of $S$ relative to paths with fixed endpoints, i.e., if $\mathrm{d} S(\delta q)=0$ for all vertical variations $\delta q$ with $\delta q(0)=0$ and $\delta q(1)=0$.

Remark 2.18. Since $\mathcal{P}_{V}(Q)_{x}=\mathcal{P}\left(Q_{x}\right)$ and $V_{q} \mathcal{P}_{V}(Q)=T_{q} \mathcal{P}\left(Q_{x}\right)$, this is immediately equivalent to $q \in \mathcal{P}_{V}(Q)_{x}$ satisfying the ordinary form of Hamilton's variational principle for the fiberrestricted Lagrangian $L_{x}$.

Having defined vertical versions of the symplectic and variational approaches to Lagrangian mechanics, we finally derive the corresponding Euler-Lagrange equations. Suppose that $q=$ $\left(x^{\sigma}, y^{i}\right)$ are fiber-adapted local coordinates for $Q$. Since vertical variations satisfy $\delta x^{\sigma}=0$, by definition, arbitrary fixed-endpoint variations of the action functional are given by

$$
\mathrm{d} S(\delta q)=\int_{0}^{1}\left(\frac{\partial L}{\partial y^{i}}(q, \dot{q}) \delta y^{i}+\frac{\partial L}{\partial \dot{y}^{i}}(q, \dot{q}) \delta \dot{y}^{i}\right) \mathrm{d} t=\int_{0}^{1}\left(\frac{\partial L}{\partial y^{i}}(q, \dot{q})-\frac{\mathrm{d}}{\mathrm{d} t} \frac{\partial L}{\partial \dot{y}^{i}}(q, \dot{q})\right) \delta y^{i} \mathrm{~d} t .
$$

Therefore, a critical vertical path must have the integrand above vanish, in addition to the vertical path condition. This motivates the following definition.

Definition 2.19. In fiber-adapted local coordinates $q=\left(x^{\sigma}, y^{i}\right)$ on $Q \rightarrow M$, the vertical Euler-Lagrange equations for $L: V Q \rightarrow \mathbb{R}$ are

$$
\dot{x}^{\sigma}=0, \quad \frac{\partial L}{\partial y^{i}}(q, \dot{q})-\frac{\mathrm{d}}{\mathrm{d} t} \frac{\partial L}{\partial \dot{y}^{i}}(q, \dot{q})=0 .
$$

Remark 2.20. Since $q=\left(x^{\sigma}, y^{i}\right)$ are fiber-adapted local coordinates, $y^{i}$ gives local coordinates for the fiber $Q_{x}$, and we may write $L(q, \dot{q})=L_{x}(y, \dot{y})$. (Note that $L$ is defined only on vertical tangent vectors, so $\dot{x}$ is not required.) Therefore, the vertical Euler-Lagrange equations are equivalent to the ordinary Euler-Lagrange equations,

$$
\frac{\partial L_{x}}{\partial y^{i}}(y, \dot{y})-\frac{\mathrm{d}}{\mathrm{d} t} \frac{\partial L_{x}}{\partial \dot{y}^{i}}(y, \dot{y})=0,
$$

for the fiber-restricted Lagrangian $L_{x}$. 
We are now prepared to state the generalization of Theorem 2.1 to Lagrangian mechanics on fibered manifolds.

Theorem 2.21. If $L: V Q \rightarrow \mathbb{R}$ is a regular Lagrangian on a fibered manifold $\mu: Q \rightarrow M$, and $q \in \mathcal{P}_{V}(Q)$ is a vertical $C^{2}$ path over $x \in M$, then the following are equivalent:

(i) $q$ is a base integral curve of the Lagrangian vector field $X_{L} \in \mathfrak{X}_{V}(V Q)$.

(ii) q satisfies Hamilton's variational principle for vertical paths.

(iii) $q$ satisfies the vertical Euler-Lagrange equations.

$\left(i^{\prime}\right) q$ is a base integral curve of the fiber-restricted Lagrangian vector field $X_{L_{x}} \in \mathfrak{X}\left(T Q_{x}\right)$.

$\left(i i^{\prime}\right)$ q satisfies Hamilton's variational principle with respect to the fiber-restricted Lagrangian $L_{x}$.

$\left(\right.$ iii $\left.{ }^{\prime}\right) q$ satisfies the Euler-Lagrange equations with respect to the fiber-restricted Lagrangian $L_{x}$. If regularity is dropped, then $(i i) \Leftrightarrow(i i i) \Leftrightarrow\left(i i^{\prime}\right) \Leftrightarrow\left(i i i^{\prime}\right)$ still holds.

Proof. We have seen, in the foregoing discussion, that $(i) \Leftrightarrow\left(i^{\prime}\right)$ for regular Lagrangians, while $(i i) \Leftrightarrow\left(i i^{\prime}\right)$ and $(i i i) \Leftrightarrow\left(i i i^{\prime}\right)$ hold in general. Hence, it suffices to show $\left(i^{\prime}\right) \Leftrightarrow\left(i i^{\prime}\right) \Leftrightarrow\left(i i i^{\prime}\right)$ for the regular case and $\left(i i^{\prime}\right) \Leftrightarrow\left(i i i^{\prime}\right)$ for the general case - but this is simply Theorem 2.1 applied to $L_{x}$.

\subsection{Application: classical Routh reduction as fibered mechanics}

The technique known as Routh reduction traces its origins as far back as the 1860 treatise of Routh [28]. Modern geometric accounts have been given by Arnold et al. [1], Marsden and Scheurle [20], and Marsden et al. [19], with the latter two works developing a more general theory of nonabelian Routh reduction.

The essence of Routh reduction, as we will show, is that it passes from a Lagrangian on an ordinary manifold to an equivalent Lagrangian, known as the Routhian, on a fibered manifold. Since the resulting dynamics are confined to the vertical components (i.e., restricted to individual fibers), this reduces the size of the original system by eliminating the horizontal components.

Consider a configuration manifold of the form $\mathbb{T}^{n} \times \mathcal{S}$, where $\mathbb{T}^{n}$ denotes the $n$-torus and $\mathcal{S}$ is a manifold called the shape space. Let $\theta^{\sigma}$ and $y^{i}$ be local coordinates for $\mathbb{T}^{n}$ and $\mathcal{S}$, respectively, and suppose the Lagrangian $L: T\left(\mathbb{T}^{n} \times S\right) \rightarrow \mathbb{R}$ is cyclic in the variables $\theta^{\sigma}$, i.e., $L=L(\dot{\theta}, y, \dot{y})$ depends only on $\dot{\theta}$ but not on $\theta$ itself. Then the $\theta^{\sigma}$ components of the Euler-Lagrange equations imply that

$$
\frac{\mathrm{d}}{\mathrm{d} t} \frac{\partial L}{\partial \dot{\theta}^{\sigma}}=\frac{\partial L}{\partial \theta^{\sigma}}=0
$$

so $x_{\sigma}=\partial L / \partial \dot{\theta}^{\sigma}$ is constant in $t$.

Now, define the fibered manifold $Q=\mathbb{R}^{n} \times \mathcal{S}$ with $M=\mathbb{R}^{n}$, where $\mu: Q \rightarrow M$ is simply the projection onto the $\mathbb{R}^{n}$ component, so that $V Q=\mathbb{R}^{n} \times T \mathcal{S}$. The classical Routhian $R: V Q \rightarrow \mathbb{R}$ is

$$
R(x, y, \dot{y})=\left[L(\dot{\theta}, y, \dot{y})-x_{\sigma} \dot{\theta}^{\sigma}\right]_{x_{\sigma}=\partial L / \partial \dot{\theta}^{\sigma}}
$$

where each $\dot{\theta}^{\sigma}$ is determined implicitly by the constraint $x_{\sigma}=\partial L / \partial \dot{\theta}^{\sigma}$. Considering $R$ as a Lagrangian in the sense of the previous section, the vertical Euler-Lagrange equations consist of the vertical path condition,

$$
0=\dot{x}_{\sigma}=\frac{\mathrm{d}}{\mathrm{d} t} \frac{\partial L}{\partial \dot{\theta}^{\sigma}}
$$


and

$$
\begin{aligned}
0 & =\frac{\partial R}{\partial y^{i}}(x, y, \dot{y})-\frac{\mathrm{d}}{\mathrm{d} t} \frac{\partial R}{\partial \dot{y}^{i}}(x, y, \dot{y}) \\
& =\left(\frac{\partial L}{\partial y^{i}}+\frac{\partial L}{\partial \dot{\theta}^{\sigma}} \frac{\partial \dot{\theta}^{\sigma}}{\partial y^{i}}-x_{\sigma} \frac{\partial \dot{\theta}^{\sigma}}{\partial y^{i}}\right)-\frac{\mathrm{d}}{\mathrm{d} t}\left(\frac{\partial L}{\partial \dot{y}^{i}}+\frac{\partial L}{\partial \dot{\theta}^{\sigma}} \frac{\partial \dot{\theta}^{\sigma}}{\partial \dot{y}^{i}}-x_{\sigma} \frac{\partial \dot{\theta}}{\partial \dot{y}^{i}}\right) \\
& =\frac{\partial L}{\partial y^{i}}-\frac{\mathrm{d}}{\mathrm{d} t} \frac{\partial L}{\partial \dot{y}^{i}},
\end{aligned}
$$

where the last step uses $x_{\sigma}=\partial L / \partial \dot{\theta}^{\sigma}$ to eliminate the last two terms from each parenthetical expression.

Thus, the ordinary Euler-Lagrange equations for $L$ are precisely equivalent to the vertical Euler-Lagrange equations for $R$. This reduces the dynamics from $\mathbb{T}^{n} \times \mathcal{S}$ to those on the individual fibers $Q_{x} \cong \mathcal{S}$, thereby eliminating the cyclic variables $\theta \in \mathbb{T}^{n}$. We now summarize this result as a theorem.

Theorem 2.22. Suppose $L: T\left(\mathbb{T}^{n} \times \mathcal{S}\right) \rightarrow \mathbb{R}$ is an ordinary Lagrangian that is cyclic in the $\mathbb{T}^{n}$ components, and let the classical Routhian $R: V\left(\mathbb{R}^{n} \times \mathcal{S}\right) \rightarrow \mathbb{R}$ be the fibered Lagrangian defined in (2.2). Then $(\theta, y) \in \mathcal{P}\left(\mathbb{T}^{n} \times S\right)$ is a solution path for $L$ if and only if $(x, y) \in \mathcal{P}_{V}\left(\mathbb{R}^{n} \times \mathcal{S}\right)$ is a vertical solution path for $R$.

Proof. This follows from Theorem 2.21, together with the foregoing calculations.

\section{Lagrangian mechanics on Lie algebroids}

In this section, we lay the groundwork for reduction theory on fibered manifolds, which will be discussed in Section 4. In ordinary Lagrangian reduction, we pass from the tangent bundle $T Q$ to the quotient $T Q / G$, which is generally not a tangent bundle. Likewise, in Section 4 , we will pass from vertical bundles to quotients that are generally not vertical bundles. However, $T Q$ and $T Q / G$ - as well as their vertical analogs, as we will show - are all examples of more general objects called Lie algebroids, on which Lagrangian mechanics can be studied. The study of Lagrangian mechanics on Lie algebroids was largely pioneered by Weinstein [30], and important follow-up work was done by Martínez [23, 24, 25] and several others in more recent years; see also Cortés et al. [4], Cortés and Martínez [5], Grabowska and Grabowski [9], Grabowska et al. [10], Iglesias et al. [12, 13].

In addition to recalling some of the key results (particularly of Weinstein [30] and Martínez [23]) that we will need for the subsequent reduction theory, we also develop a new, coordinate-free formulation of the equations of motion, which we call the Euler-Lagrange-Poincaré equations (since they simultaneously generalize the Euler-Lagrange, Euler-Poincaré, and Lagrange-Poincaré equations). This new formulation is based on the work of Crainic and Fernandes [6], particularly the notion of a Lie algebroid connection and its use in describing variations of paths.

\subsection{Lie algebroids and $A$-paths}

We begin by recalling the definition of a Lie algebroid $A$ and an appropriate class of paths in $A$, called $A$-paths. This review will necessarily be very brief, but for more information on Lie algebroids, we refer the reader to the comprehensive work by Mackenzie [14].

Definition 3.1. A Lie algebroid is a real vector bundle $\tau: A \rightarrow Q$ equipped with a Lie bracket $[\cdot, \cdot]: \Gamma(A) \times \Gamma(A) \rightarrow \Gamma(A)$ on its space of sections and a bundle map $\rho: A \rightarrow T Q$, called the anchor map, satisfying the following Leibniz rule-like compatibility condition:

$$
[X, f Y]=f[X, Y]+\rho(X)[f] Y, \quad \text { for all } X, Y \in \Gamma(A), f \in C^{\infty}(Q) .
$$


Example 3.2. The tangent bundle $T Q$ is a Lie algebroid over $Q$, where $\tau: T Q \rightarrow Q$ is the usual bundle projection, $[\cdot, \cdot]: \mathfrak{X}(Q) \times \mathfrak{X}(Q) \rightarrow \mathfrak{X}(Q)$ is the Jacobi-Lie bracket of vector fields, and $\rho: T Q \rightarrow T Q$ is the identity.

Furthermore, any integrable distribution $\mathcal{D} \subset T Q$ is also a Lie algebroid over $Q$, where $\tau,[\cdot, \cdot]$, and $\rho$ are just the restrictions to $\mathcal{D}$ of the corresponding maps for $T Q$. We say that $\mathcal{D}$ is a Lie subalgebroid of $T Q$.

In particular, if $Q \rightarrow M$ is a fibered manifold, then $V Q \subset T Q$ is integrable and hence a Lie algebroid over $Q$. (Note that $V Q$ is generally not a Lie algebroid over $M$, since it may not even be a vector bundle over $M$.)

Example 3.3. Any Lie algebra $\mathfrak{g}$ is a Lie algebroid over $\bullet$ (the space with one point), where the maps $\tau$ and $\rho$ are trivial and $[\cdot, \cdot]$ is the Lie bracket.

More generally, if $Q \rightarrow Q / G$ is a principal $G$-bundle for some Lie group $G$, then $T Q / G$ defines an algebroid over $Q / G$ called the Atiyah algebroid. The algebroid $\mathfrak{g} \rightarrow \bullet$ can be identified with the special case $Q=G$, where $G$ is the Lie group integrating $\mathfrak{g}$ (which exists by Lie's third theorem).

Definition 3.4. A path $a \in \mathcal{P}(A)$ over the base path $q=\tau \circ a \in \mathcal{P}(Q)$ is called an $A$-path if $\dot{q}(t)=\rho(a(t))$ for all $t \in I$. The space of $A$-paths is denoted by $\mathcal{P}_{\rho}(A)$.

Remark 3.5. Equivalently, $a$ is an $A$-path if and only if $a \mathrm{~d} t: T I \rightarrow A$ is a morphism of Lie algebroids, where $T I \rightarrow I$ has the tangent bundle Lie algebroid structure of Example 3.2. Hence, $A$-paths can be seen as "paths in the category of Lie algebroids".

\subsection{Connections and variations of $A$-paths}

We now turn to discussing an appropriate class of variations on the space of $A$-paths, $\mathcal{P}_{\rho}(A)$. Crainic and Fernandes [6, Lemma 4.6] show that $\mathcal{P}_{\rho}(A) \subset \mathcal{P}(A)$ is a Banach submanifold. However, we do not want to take arbitrary variations $\delta a \in T_{a} \mathcal{P}_{\rho}(A)$, just as we did not want to take arbitrary paths in $\mathcal{P}(A)$.

To illustrate the reasoning behind this, consider the case of a Lie algebra $\mathfrak{g}$. Since this is a Lie algebroid over $\bullet$, where $\tau$ and $\rho$ are trivial, it follows that every path $\xi \in \mathcal{P}(\mathfrak{g})$ is a $\mathfrak{g}$-path, i.e., $\mathcal{P}_{\rho}(\mathfrak{g})=\mathcal{P}(\mathfrak{g})$. However, the variational principle for the Euler-Poincaré equations on $\mathfrak{g}$ considers only variations of the form

$$
\delta \xi=[\xi, \eta]+\dot{\eta}=\operatorname{ad}_{\xi} \eta+\dot{\eta},
$$

where $\eta \in \mathcal{P}(\mathfrak{g})$ is an arbitrary path vanishing at the endpoints (cf. Marsden and Ratiu [18, Chapter 13]). These constraints on admissible variations are known as Lin constraints.

To generalize these constrained variations to an arbitrary Lie algebroid $A \rightarrow Q$, we first discuss the notion of a connection on a Lie algebroid, of which the adjoint action $(\xi, \eta) \mapsto \operatorname{ad}_{\xi} \eta$ of $\mathfrak{g}$ on itself will be a special case.

Definition 3.6. If $A \rightarrow Q$ is a Lie algebroid and $E \rightarrow Q$ is a vector bundle, then an $A$-connection on $E$ is a bilinear map $\nabla: \Gamma(A) \times \Gamma(E) \rightarrow \Gamma(E),(X, u) \mapsto \nabla_{X} u$, satisfying the conditions

$$
\nabla_{f X} u=f \nabla_{X} u, \quad \nabla_{X}(f u)=f \nabla_{X} u+\rho(X)[f] u,
$$

for all $X \in \Gamma(A), u \in \Gamma(E)$, and $f \in C^{\infty}(Q)$.

Remark 3.7. A $T Q$-connection is just an ordinary connection. Given a $T Q$-connection $\nabla$ on $A$, there are two naturally-induced $A$-connections on $A$, which we write as $\nabla$ and $\bar{\nabla}$ :

$$
\nabla_{X} Y=\nabla_{\rho(X)} Y, \quad \bar{\nabla}_{X} Y=\nabla_{\rho(Y)} X+[X, Y] .
$$


For example, when $A=\mathfrak{g} \rightarrow \bullet$, the trivial $T \bullet$-connection induces two $\mathfrak{g}$-connections on $\mathfrak{g}$ :

$$
\nabla_{X} Y=0, \quad \bar{\nabla}_{X} Y=[X, Y]=\operatorname{ad}_{X} Y .
$$

Hence, the induced connection $\bar{\nabla}$ can be seen as a generalization of the adjoint action of a Lie algebra.

Definition 3.8. Let $a \in \mathcal{P}_{\rho}(A)$ be an $A$-path over $q \in \mathcal{P}(Q)$ and $\xi \in \mathcal{P}(\Gamma(A))$ be a timedependent section such that $a(t)=\xi(q(t))$. Suppose $u \in \mathcal{P}(E)$ has the same base path $q$, along with a time-dependent section $\eta \in \mathcal{P}(\Gamma(E))$ satisfying $u(t)=\eta(q(t))$. Then we define

$$
\nabla_{a} u(t)=\nabla_{\xi} \eta(t, q(t))+\dot{\eta}(t, q(t)),
$$

which is independent of the choice of $\xi, \eta$.

Definition 3.9. Let $a \in \mathcal{P}_{\rho}(A)$ be an $A$-path over $q \in \mathcal{P}(Q)$. An admissible variation of $a$ is a variation of the form $X_{b, a} \in T_{a} \mathcal{P}_{\rho}(A)$, where $b \in \mathcal{P}(A)$ is a path in $A$ (but not necessarily an $A$-path!) over $q$ such that $b(0)=0$ and $b(1)=0$. Relative to a $T Q$-connection $\nabla$, the variation $X_{b, a}$ has vertical component $\bar{\nabla}_{a} b$ and horizontal component $\rho(b)$.

Remark 3.10. Crainic and Fernandes [6, Proposition 4.7] show that these admissible variations form an integrable subbundle $\mathcal{F}(A) \subset T \mathcal{P}_{\rho}(A)$, and the tangent subspaces $\mathcal{F}_{a}(A) \subset T_{a} \mathcal{P}_{\rho}(A)$ are independent of the choice of connection $\nabla$ in the above definition.

\subsection{Lagrangian mechanics}

Now that we have appropriate paths and variations, we are prepared to discuss the variational approach to Lagrangian mechanics on Lie algebroids.

Definition 3.11. Given a Lagrangian $L: A \rightarrow \mathbb{R}$, the action functional $S: \mathcal{P}_{\rho}(A) \rightarrow \mathbb{R}$ is defined to be

$$
S(a)=\int_{0}^{1} L(a(t)) \mathrm{d} t .
$$

We say that $a \in \mathcal{P}_{\rho}(A)$ satisfies Hamilton's variational principle for $A$-paths if $\mathrm{d} S\left(X_{b, a}\right)=0$ for all admissible variations $X_{b, a} \in \mathcal{F}_{a}(A)$.

We next use the notion of admissible variation from Definition 3.9, and its expression in terms of a connection on $A$, to give a new, coordinate-free characterization of the solutions to Hamilton's variational principle for $A$-paths.

Theorem 3.12. An A-path $a \in \mathcal{P}_{\rho}(A)$ satisfies Hamilton's principle if and only if, given a TQconnection $\nabla$ on $A$, it satisfies the differential equation

$$
\rho^{*} \mathrm{~d} L^{\text {hor }}(a)+\bar{\nabla}_{a}^{*} \mathrm{~d} L^{\mathrm{ver}}(a)=0,
$$

where $\mathrm{d} L^{\text {hor }}$ and $\mathrm{d} L^{\text {ver }}$ are the horizontal and vertical components of $\mathrm{d} L$ relative to $\nabla$, and where $\rho^{*}$ and $\bar{\nabla}_{a}^{*}$ are the formal adjoints of $\rho$ and $\bar{\nabla}_{a}$.

Proof. Given an admissible variation $X_{b, a} \in \mathcal{F}_{a}(A)$, we have

$$
\begin{aligned}
\mathrm{d} S\left(X_{b, a}\right) & =\mathrm{d} S\left(X_{b, a}^{\mathrm{hor}}\right)+\mathrm{d} S\left(X_{b, a}^{\mathrm{ver}}\right)=\int_{0}^{1}\left(\left\langle\mathrm{~d} L^{\mathrm{hor}}(a), \rho(b)\right\rangle+\left\langle\mathrm{d} L^{\mathrm{ver}}(a), \bar{\nabla}_{a} b\right\rangle\right) \mathrm{d} t \\
& =\int_{0}^{1}\left\langle\rho^{*} \mathrm{~d} L^{\text {hor }}(a)+\bar{\nabla}_{a}^{*} \mathrm{~d} L^{\mathrm{ver}}(a), b\right\rangle \mathrm{d} t
\end{aligned}
$$

Since $b$ is arbitrary, it follows that $\mathrm{d} S$ vanishes for all $X_{b, a} \in \mathcal{F}_{a}(A)$ if and only if $\rho^{*} \mathrm{~d} L^{\text {hor }}(a)+$ $\bar{\nabla}_{a}^{*} \mathrm{~d} L^{\mathrm{ver}}(a)$ vanishes for all $t$. 
Example 3.13. Let $A=\mathfrak{g} \rightarrow \bullet$, where $\mathfrak{g}$ is a Lie algebra. Any $a \in \mathcal{P}_{\rho}(\mathfrak{g})=\mathcal{P}(\mathfrak{g})$ can be identified with its unique time-dependent section $\xi(t)=\xi(t, \bullet)=a(t)$. Since $\rho$ and $\nabla$ are trivial, it follows that (3.1) becomes

$$
0=\bar{\nabla}_{a}^{*} \mathrm{~d} L(a)=\left(\operatorname{ad}_{\xi}+\frac{\mathrm{d}}{\mathrm{d} t}\right)^{*} \frac{\delta L}{\delta \xi}=\left(\operatorname{ad}_{\xi}^{*}-\frac{\mathrm{d}}{\mathrm{d} t}\right) \frac{\delta L}{\delta \xi},
$$

which are precisely the Euler-Poincaré equations (cf. Marsden and Ratiu [18, Chapter 13]).

Next, we show that this coordinate-free formulation agrees with the local-coordinate expression obtained by Weinstein [30] for regular Lagrangians and by Martínez [23, 24, 25] in the more general case.

Theorem 3.14. Let $q^{i}$ be local coordinates for $Q,\left\{e_{I}\right\}$ be a local basis of sections of $A$, and $\nabla$ the locally trivial TQ-connection defined by $\nabla_{\partial / \partial q^{i}} e_{I} \equiv 0$. Let $\rho_{I}^{i}$ and $C_{I J}^{K}$ be the local-coordinate representations of $\rho$ and $[\cdot, \cdot]$, where

$$
\rho\left(e_{I}\right)=\rho_{I}^{i} \frac{\partial}{\partial q^{i}}, \quad\left[e_{I}, e_{J}\right]=C_{I J}^{K} e_{K}
$$

If $a \in \mathcal{P}(A)$ has the local-coordinate representation $a(t)=\xi^{I}(t) e_{I}(q(t))$, then $a$ is an $A$-path if and only if $\dot{q}^{i}=\rho_{I}^{i} \xi^{I}$, and a satisfies (3.1) if and only if

$$
\rho_{I}^{i} \frac{\partial L}{\partial q^{i}}-C_{I J}^{K} \xi^{J} \frac{\partial L}{\partial \xi^{K}}-\frac{\mathrm{d}}{\mathrm{d} t} \frac{\partial L}{\partial \xi^{I}}=0 .
$$

Proof. For the $A$-path condition, we have

$$
\dot{q}=\dot{q}^{i} \frac{\partial}{\partial q^{i}}, \quad \rho(a)=\rho\left(\xi^{I} e_{I}\right)=\rho_{I}^{i} \xi^{I} \frac{\partial}{\partial q^{i}},
$$

so these are equal if and only the $\partial / \partial q^{i}$ coefficients are equal. Next, the horizontal and vertical components of $\mathrm{d} L$ are

$$
\mathrm{d} L^{\text {hor }}=\frac{\partial L}{\partial q^{i}} \mathrm{~d} q^{i}, \quad \mathrm{~d} L^{\mathrm{ver}}=\frac{\partial L}{\partial \xi^{I}} e^{I},
$$

where, as usual, $e^{I}$ is the dual basis element satisfying $e^{I} e_{J}=\delta_{J}^{I}$. Moreover, extending $a$ to the time-dependent section $\xi(t)=\xi^{J}(t) e_{J}$, we have

$$
\bar{\nabla}_{a} \eta^{I} e_{I}=\bar{\nabla}_{\xi^{J} e_{J}} \eta^{I} e_{I}+\dot{\eta}^{I} e_{I}=\left[\xi^{J} e_{J}, \eta^{I} e_{I}\right]+\dot{\eta}^{I} e_{I}=-C_{I J}^{K} \xi^{J} \eta^{I} e_{K}+\dot{\eta}^{I} e_{I},
$$

so $\bar{\nabla}_{a}=-C_{I J}^{K} \xi^{J} e^{I} e_{K}+\mathrm{d} / \mathrm{d} t$. Finally,

$$
\rho^{*} \mathrm{~d} L^{\mathrm{hor}}+\bar{\nabla}_{a}^{*} \mathrm{~d} L^{\mathrm{ver}}=\rho_{I}^{i} \frac{\partial L}{\partial q^{i}} e^{I}-C_{I J}^{K} \xi^{J} \frac{\partial L}{\partial \xi^{K}} e^{I}-\frac{\mathrm{d}}{\mathrm{d} t} \frac{\partial L}{\partial \xi^{I}} e^{I},
$$

so the left side vanishes if and only if all the $e^{I}$ coefficients on the right side vanish, i.e., (3.1) holds if and only if (3.2) holds.

Example 3.15. Suppose $A=T Q \rightarrow Q$. Local coordinates $q^{i}$ on $Q$ yield corresponding local sections $\partial / \partial q^{i}$ of $T Q$, i.e., $e_{i}=\partial / \partial q^{i}$. It follows that $\left[e_{i}, e_{j}\right] \equiv 0$ and thus $C_{i j}^{k} \equiv 0$ for all $i, j, k$. Since $\rho$ is the identity map, we have $\rho_{j}^{i}=\delta_{j}^{i}$, so the $T Q$-path condition is $\dot{q}^{i}=\xi^{i}$. Putting this all together, it follows that (3.2) yields

$$
\frac{\partial L}{\partial q^{i}}-\frac{\mathrm{d}}{\mathrm{d} t} \frac{\partial L}{\partial \dot{q}^{i}}=0
$$

i.e., the ordinary Euler-Lagrange equations. 
Remark 3.16. There is also an equivalent symplectic/pre-symplectic/Poisson approach to Lagrangian mechanics on Lie algebroids, which has already been well studied in previous work on the subject.

Martínez [23] shows that one can define a Lie algebroid notion of differential forms (just as we did for the vertical formalism in Section 2.2), as well as a version of the tautological 1-form and canonical 2-form on $A^{*}$. The Legendre transform $\mathbb{F} L=\mathrm{d} L^{\mathrm{ver}}: A \rightarrow A^{*}$ is then used to pull this back to a Lagrangian 2-form on $A$ (in the sense of forms on Lie algebroids) and to define an energy function $E_{L}$ on $A$, which Martínez [23] uses to obtain Lagrangian dynamics on $A$.

Weinstein [30], on the other hand, uses the canonical Poisson structure on $A^{*}$ (which generalizes the Lie-Poisson structure on the dual of a Lie algebra), which can be pulled back along $\mathbb{F} L$ to $A$ when $L$ is a regular Lagrangian. In this case, the Poisson structure on $A$ induces a Lagrangian vector field associated to $E_{L}$ in the usual way.

The approach of Grabowska et al. [10], Grabowska and Grabowski [9] extends Weinstein's approach in a different direction: instead of using the canonical Poisson structure on $A^{*}$, which maps $T^{*} A^{*} \rightarrow T A^{*}$, they use a related map $\epsilon: T^{*} A \rightarrow T A^{*}$ to define the Tulczyjew differential $\Lambda_{L}=\epsilon \circ \mathrm{d} L: A \rightarrow T A^{*}$. (The map $\epsilon$ is related to the canonical Poisson map by the Tulczyjew isomorphism $T^{*} A^{*} \cong T^{*} A$.) Using this framework, one requires that $a \in \mathcal{P}(A)$ satisfy $\frac{\mathrm{d}}{\mathrm{d} t} \mathbb{F} L(a)=\Lambda_{L}(a)$, which contains the Euler-Lagrange-Poincaré equations together with the $A$-path condition. We remark that Grabowska et al. [10], Grabowska and Grabowski [9] apply this approach both to Lie algebroids and to so-called "general algebroids," for which the map $\epsilon$ is taken as primitive, and where there is generally no canonical Poisson structure on the dual.

\subsection{Special case: the Lagrange-Poincaré equations}

The Lagrange-Poincaré equations on a principal bundle $Q \rightarrow Q / G$ are typically derived by the procedure of Lagrangian reduction (cf. Marsden and Scheurle [21], Cendra et al. [3]), relative to a particular choice of principal connection. We now discuss how these equations may instead be obtained directly on the Atiyah algebroid $A=T Q / G \rightarrow Q / G$, using the framework presented above, and how the choice of principal connection is related to the connection $\nabla$ on $A$. (Note that $Q / G$, not $Q$, is the base of this algebroid.) In particular, Example 3.13 corresponds to the case $Q=G$, while Example 3.15 corresponds to the case where $G$ is trivial.

Let $L: T Q / G \rightarrow \mathbb{R}$ be a Lagrangian on the Atiyah algebroid. A principal connection corresponds to a section of the anchor $\rho: T Q / G \rightarrow T(Q / G)$, i.e., a right splitting of the Atiyah sequence,

$$
0 \rightarrow \widetilde{\mathfrak{g}} \rightarrow T Q / G \stackrel{\rho}{\rightarrow} T(Q / G) \rightarrow 0 .
$$

Here, following Cendra et al. [3], we use $\widetilde{\mathfrak{g}}$ to denote the adjoint bundle $Q \times_{G} \mathfrak{g}$, so a left splitting is a principal connection 1-form (cf. Mackenzie [14, Chapter 5]). This splitting lets us write $T Q / G \cong T(Q / G) \oplus \tilde{\mathfrak{g}}$; the anchor $\rho$ is just projection onto the first component, and the bracket of two sections $\xi=(X, \bar{\xi})$ and $\eta=(Y, \bar{\eta})$ is

$$
[(X, \bar{\xi}),(Y, \bar{\eta})]=\left([X, Y], \widetilde{\nabla}_{X} \bar{\eta}-\widetilde{\nabla}_{Y} \bar{\xi}+[\bar{\xi}, \bar{\eta}]-\widetilde{R}(X, Y)\right)
$$

where $\widetilde{\nabla}$ is the covariant derivative and $\widetilde{R}$ the curvature form of the principal connection (cf. Cendra et al. [3, Theorem 5.2.4] in this particular case and Mackenzie [14, Theorem 7.3.7] in a more general setting).

Relative to the splitting induced by the principal connection, $A$-paths have the form $a=$ $(x, \dot{x}, \bar{v})$, where $x$ is the base path in $Q / G$. As before, we extend $a$ to a time-dependent section $\xi=(X, \bar{\xi})$, and likewise, we extend an arbitrary path $b=(x, \delta x, \bar{w})$ to a time-dependent section 
$\eta=(Y, \bar{\eta})$. To find the corresponding admissible variation $\delta a$, we calculate $\rho(b)=\delta x$ and use (3.4) to obtain

$$
\begin{aligned}
\bar{\nabla}_{a} b & =\bar{\nabla}_{\xi} \eta+\dot{\eta}=\bar{\nabla}_{(X, \bar{\xi})}(Y, \bar{\eta})+(\dot{Y}, \dot{\bar{\eta}})=\nabla_{Y}(X, \bar{\xi})+[(X, \bar{\xi}),(Y, \bar{\eta})]+(\dot{Y}, \dot{\bar{\eta}}) \\
& =\left(\nabla_{Y} X+[X, Y]+\dot{Y}, \widetilde{\nabla}_{X} \bar{\eta}+[\bar{\xi}, \bar{\eta}]-\widetilde{R}(X, Y)+\dot{\bar{\eta}}\right) \\
& =\left(\bar{\nabla}_{X} Y+\dot{Y},\left(\widetilde{\nabla}_{X} \bar{\eta}+\dot{\bar{\eta}}\right)+[\bar{\xi}, \bar{\eta}]-\widetilde{R}(X, Y)\right) \\
& =\left(\bar{\nabla}_{\dot{x}}(\delta x), \widetilde{\nabla}_{\dot{x}} \bar{w}+[\bar{v}, \bar{w}]-\widetilde{R}(\dot{x}, \delta x)\right) .
\end{aligned}
$$

(Here, we chose $\nabla$ to be compatible with $\widetilde{\nabla}$, so that the $\nabla_{Y} \bar{\xi}$ and $\widetilde{\nabla}_{Y} \bar{\xi}$ terms cancel.) Therefore, admissible variations have the form $\delta a=(\delta x, \delta \dot{x}, \delta \bar{v})$, where

$$
\delta \bar{v}=\widetilde{\nabla}_{\dot{x}} \bar{w}+[\bar{v}, \bar{w}]-\widetilde{R}(\dot{x}, \delta x),
$$

and these are precisely the admissible variations of Cendra et al. [3, Theorem 3.4.1].

Furthermore, now that we have expressions for $\rho$ and $\bar{\nabla}$ in terms of the splitting induced by the principal connection, it is a straightforward matter to write down the Euler-Lagrange-Poincaré equations (3.1) in terms of their adjoints. If we write $L=L(x, \dot{x}, \bar{v})$, then

$$
\begin{aligned}
& \rho^{*} \mathrm{~d} L^{\text {hor }}(x, \dot{x}, \bar{v})+\bar{\nabla}_{(x, \dot{x}, \bar{v})}^{*} \mathrm{~d} L^{\text {ver }}(x, \dot{x}, \bar{v}) \\
& \quad=\left(\frac{\partial L}{\partial x}+\bar{\nabla}_{\dot{x}}^{*} \frac{\partial L}{\partial \dot{x}}-\left(i_{\dot{x}} \widetilde{R}\right)^{*} \frac{\partial L}{\partial \bar{v}}\right) \mathrm{d} x+\left(\widetilde{\nabla}_{\dot{x}}^{*} \frac{\partial L}{\partial \bar{v}}+\operatorname{ad}_{\bar{v}}^{*} \frac{\partial L}{\partial \bar{v}}\right) \mathrm{d} \bar{v} .
\end{aligned}
$$

Hence, this vanishes precisely when

$$
\frac{\partial L}{\partial x}+\bar{\nabla}_{\dot{x}}^{*} \frac{\partial L}{\partial \dot{x}}-\left(i_{\dot{x}} \widetilde{R}\right)^{*} \frac{\partial L}{\partial \bar{v}}=0, \quad \widetilde{\nabla}_{\dot{x}}^{*} \frac{\partial L}{\partial \bar{v}}+\operatorname{ad}_{\bar{v}}^{*} \frac{\partial L}{\partial \bar{v}}=0,
$$

which are exactly the coordinate-free Lagrange-Poincaré equations of Cendra et al. [3, Theorem 3.4.1]. (The only notable difference in notation is that Cendra et al. [3] write both covariant derivatives $\bar{\nabla}_{\dot{x}}$ and $\widetilde{\nabla}_{\dot{x}}$ as $D / D t$ and their adjoints as $-D / D t$.)

Remark 3.17. The argument above works not only for the Atiyah algebroid of a principal bundle, but also in the more general setting discussed in Mackenzie [14, Chapter 7], where one can split a short exact sequence similar to (3.3) and obtain a bracket of the form (3.4). This includes the so-called transitive Lie algebroids, of which the Atiyah algebroid is a particular example.

Example 3.18. Wong's equations [31] for a particle in a Yang-Mills field are a classic example of Lagrange-Poincaré theory. Following the presentation in Cendra et al. [3, Chapter 4], we suppose that $Q \rightarrow Q / G$ is a principal $G$-bundle equipped with a Riemannian metric $g$ on the base $Q / G$ and a bi-invariant Riemannian metric $\kappa$ on the structure group $G$. Using a principal connection to split $T Q / G \cong T(Q / G) \oplus \widetilde{\mathfrak{g}}$, and denoting by $k$ the fiber metric on $\widetilde{\mathfrak{g}}$ corresponding to $\kappa$, we take the Lagrangian

$$
L(x, \dot{x}, \bar{v})=\frac{1}{2} k(\bar{v}, \bar{v})+\frac{1}{2} g(\dot{x}, \dot{x}) .
$$

The affine connection $\nabla$ is then chosen to agree with $\widetilde{\nabla}$ on $\widetilde{\mathfrak{g}}$ and with the Levi-Civita connection associated to $g$ on the base.

With this connection in hand, we now compute the $\mathrm{d} L^{\mathrm{ver}}$ components,

$$
\frac{\partial L}{\partial \dot{x}}=g(\dot{x}, \cdot)=g^{b}(\dot{x}), \quad \frac{\partial L}{\partial \bar{v}}=k(\bar{v}, \cdot)=k^{b}(\bar{v}),
$$


using the familiar "flat" notation for metrics. Since the fiber metric $k$ is necessarily ad-invariant, the term $\operatorname{ad}_{\bar{v}}^{*} k^{b}(\bar{v})$ vanishes, so the $\mathrm{d} \bar{v}$ component of the Lagrange-Poincaré equations is

$$
\widetilde{\nabla}_{\dot{x}} k^{b}(\bar{v})=0 .
$$

Next, since $\nabla$ agrees with the Levi-Civita connection on $Q / G$, the torsion-free property implies

$$
\bar{\nabla}_{X} Y=\nabla_{Y} X+[X, Y]=\nabla_{X} Y
$$

so we just have $\bar{\nabla} \equiv \nabla$. Moreover, using the metric-compatibility of $\nabla$ along with (3.5) to compute $\mathrm{d} L^{\text {hor }}$, it can be seen that

$$
\frac{\partial L}{\partial x}+\bar{\nabla}_{\dot{x}}^{*} \frac{\partial L}{\partial \dot{x}}=g^{b}\left(\nabla_{\dot{x}} \dot{x}\right),
$$

and therefore the $\mathrm{d} x$ component of the Lagrange-Poincaré equations is

$$
g^{b}\left(\nabla_{\dot{x}} \dot{x}\right)=\left(i_{\dot{x}} \widetilde{R}\right)^{*} k^{b}(\bar{v}) .
$$

The equations (3.5) and (3.6) are precisely the coordinate-free version of Wong's equations. For further discussion on Wong's equations from the perspective of Lie algebroids, see León et al. [7], Grabowska et al. [10].

We conclude this example with some remarks on the relationship between Wong's equations and the generalized notion of geodesics on a Lie algebroid. Montgomery [26] called $g \oplus k$ a KaluzaKlein metric and related Wong's equations to Kaluza-Klein geodesics. However, a Kaluza-Klein metric is a particular example of a Lie algebroid metric (in this case, on $A=T Q / G$ ), for which there is a unique Levi-Civita (torsion-free, metric-compatible) $A$-connection $\nabla$, and one may consider the corresponding geodesic equations,

$$
\nabla_{a} a=0 .
$$

(See Crainic and Fernandes [6], Cortés and Martínez [5], Cortés et al. [4].) Grabowska et al. [10] pointed out that Wong's equations may in fact be considered a special case of the generalized geodesic equations on a Lie algebroid; this correspondence is hidden slightly by the fact that

Wong's equations are written relative to an $A$-connection obtained from $\tilde{\nabla}$ rather than the Levi-Civita $A$-connection.

\subsection{Fibered manifolds revisited}

The results of Section 2 for fibered manifolds are, in fact, a special case of Lagrangian mechanics on the Lie algebroid $V Q$.

Recall from Example 3.2 that, whenever $Q \rightarrow M$ is a fibered manifold, the vertical bundle $V Q$ is a Lie algebroid over $Q$; in particular, it is a Lie subalgebroid of $T Q$, from which it inherits the bracket $[\cdot, \cdot]$, projection $\rho$, and (identity) anchor $\rho$. Now, by Definition 3.4, $a \in \mathcal{P}(V Q)$ over $q \in \mathcal{P}(Q)$ is a $V Q$-path if and only if it satisfies $\dot{q}=a$. Since $a(t) \in V Q$ for each $t \in I$, this means that $V Q$-paths are precisely the tangent prolongations of vertical paths $q \in \mathcal{P}_{V}(Q)$. Hence, we may identify $\mathcal{P}_{\rho}(V Q)$ with $\mathcal{P}_{V}(Q)$.

Suppose now that $L: V Q \rightarrow \mathbb{R}$ is a Lagrangian in the sense of Section 3.3. If $\left(x^{\sigma}, y^{i}\right)$ are fiber-adapted local coordinates for $Q \rightarrow M$, then $e_{i}=\partial / \partial y^{i}$ defines a basis of local sections of $V Q$. Since an $A$-path is just a tangent prolongation of a vertical path, it follows that the $A$-path conditions are $\dot{x}^{\sigma}=0$ and $\dot{y}^{i}=\xi^{i}$. Furthermore, as in Example 3.15, we have $\rho_{j}^{i}=\delta_{j}^{i}$, $\rho_{\sigma}^{i} \equiv 0$, and $C_{i j}^{k} \equiv 0$, so (3.2) becomes

$$
\frac{\partial L}{\partial y^{i}}-\frac{\mathrm{d}}{\mathrm{d} t} \frac{\partial L}{\partial \dot{y}^{i}}=0 \text {. }
$$

Together with the $A$-path condition, this agrees precisely with the vertical Euler-Lagrange equations (2.1). 


\subsection{Lie algebroid morphisms and reduction}

Finally, we give a brief review of Lagrangian reduction on Lie algebroids. Weinstein [30] and Martínez [25] showed that, whenever $\Phi: A \rightarrow A^{\prime}$ is a Lie algebroid morphism, then one can relate Lagrangian dynamics on $A$ to those on $A^{\prime}$.

Informally, a Lie algebroid morphism is a mapping that "preserves" the Lie algebroid structure in an appropriate sense. More precisely, if $A \rightarrow M$ and $A^{\prime} \rightarrow M^{\prime}$ are Lie algebroids (possibly over different base manifolds), then a bundle mapping $\Phi: A \rightarrow A^{\prime}$ is a Lie algebroid morphism if the dual comorphism $\Phi^{*}: A^{*} \rightarrow A^{*}$ is a Poisson relation with respect to the canonical Poisson structures on $A^{*}$ and $A^{\prime *}$. (See also Remark 3.16.)

Theorem 3.19. Let $\Phi: A \rightarrow A^{\prime}$ be a morphism of Lie algebroids, and suppose $L: A \rightarrow \mathbb{R}$ and $L^{\prime}: A^{\prime} \rightarrow \mathbb{R}$ are Lagrangians such that $L=L^{\prime} \circ \Phi$. If $a \in \mathcal{P}_{\rho}(A)$ is such that $a^{\prime}=\Phi \circ a \in \mathcal{P}_{\rho^{\prime}}\left(A^{\prime}\right)$ is a solution path for $L^{\prime}$, then a is a solution path for $L$. Moreover, the following converse holds when $\Phi: A \rightarrow A^{\prime}$ is fiberwise surjective: If $a \in \mathcal{P}_{\rho}(A)$ is a solution path for $L$, then $a^{\prime}=\Phi \circ a \in \mathcal{P}_{\rho^{\prime}}\left(A^{\prime}\right)$ is a solution path for $L^{\prime}$.

Proof. See Martínez [25, Theorems 5-6]. This generalized results by Weinstein [30, Theorems 4.8 and 4.5, respectively] for regular Lagrangians, where the converse also required the stronger assumption that $\Phi$ be a fiberwise isomorphism.

For example, if $G$ is a Lie group acting freely and properly on $Q$, then the quotient morphism $T Q \rightarrow T Q / G$ is a Lie algebroid morphism, and the corresponding reduction theory is just classical Lagrangian reduction. However, there is a much more general class of quotient morphisms for fibered manifolds - that bear directly on reduction theory, and this is the topic of the next section.

\section{Lie groupoid symmetries and reduction on fibered manifolds}

In this section, we recall the definition of a Lie groupoid $G \rightrightarrows M$ and of a free, proper Lie groupoid action on a fibered manifold $Q \rightarrow M$ over the same base manifold. We then show that there is a quotient morphism $V Q \rightarrow V Q / G$, which is a Lie algebroid morphism, and hence applying Theorem 3.19 yields a reduction theory for fibered Lagrangian mechanics. This generalizes the special case $M=\bullet$, in which $G$ is a Lie group acting on an ordinary manifold $Q$ and the quotient morphism $T Q \rightarrow T Q / G$ is the one used in ordinary Lagrangian reduction.

\subsection{Lie groupoids}

Just as it is natural to consider Lie group actions on ordinary manifolds, it is natural to consider Lie groupoid actions on fibered manifolds. We begin by recalling the definition of a Lie groupoid and a groupoid action, as well as giving a few examples. We then prove that, just as a free and proper Lie group action on an ordinary manifold $Q$ lifts to $T Q$, so, too, does a free and proper Lie groupoid action on a fibered manifold $Q \rightarrow M$ lift to $V Q$.

Definition 4.1. A groupoid is a small category in which every morphism is invertible. Specifically, a groupoid denoted $G \rightrightarrows M$ consists of a space of morphisms $G$, a space of objects $M$, and the following structure maps:

(i) a source map $\alpha: G \rightarrow M$ and target map $\beta: G \rightarrow M$;

(ii) a multiplication map $m: G_{\alpha} \times{ }_{\beta} G \rightarrow G,(g, h) \mapsto g h$;

(iii) an identity section $\epsilon: M \rightarrow G$, such that for all $g \in G$,

$$
g \epsilon(\alpha(g))=g=\epsilon(\beta(g)) g
$$


(iv) and an inversion map $i: G \rightarrow G, g \mapsto g^{-1}$, such that for all $g \in G$,

$$
g^{-1} g=\epsilon(\alpha(g)), \quad g g^{-1}=\epsilon(\beta(g)) .
$$

A Lie groupoid is a groupoid $G \rightrightarrows M$ where $G$ and $M$ are smooth manifolds, $\alpha$ and $\beta$ are submersions, and $m$ is smooth.

Remark 4.2. A few other properties of the structure maps are immediate from this definition of a Lie groupoid: in particular, it also follows that $m$ is a submersion, $\epsilon$ is an immersion, and $i$ is a diffeomorphism.

Example 4.3. A Lie group is a Lie groupoid $G \rightrightarrows \bullet$ over a single point.

Example 4.4. If $Q$ is a smooth manifold, then the pair groupoid $Q \times Q \rightrightarrows Q$, defined by the structure maps

$$
\begin{aligned}
& \alpha\left(q_{1}, q_{0}\right)=q_{0}, \quad \beta\left(q_{1}, q_{0}\right)=q_{1}, \quad m\left(\left(q_{2}, q_{1}\right),\left(q_{1}, q_{0}\right)\right)=\left(q_{2}, q_{0}\right), \\
& \epsilon(q)=(q, q), \quad i\left(q_{1}, q_{0}\right)=\left(q_{0}, q_{1}\right),
\end{aligned}
$$

is a Lie groupoid. More generally, if $\mu: Q \rightarrow M$ is a fibered manifold and

$$
Q_{\mu} \times{ }_{\mu} Q=\left\{\left(q_{1}, q_{0}\right) \in Q \times Q: \mu\left(q_{1}\right)=\mu\left(q_{2}\right)\right\},
$$

then $Q{ }_{\mu} \times{ }_{\mu} Q \rightrightarrows Q$ is also a Lie groupoid, and its structure maps are just the restrictions of those above for $Q \times Q \rightrightarrows Q$. We then say that $Q_{\mu} \times{ }_{\mu} Q \rightrightarrows Q$ is a Lie subgroupoid of $Q \times Q \rightrightarrows Q$.

Example 4.5. Let $G$ be a Lie group and $Q \rightarrow Q / G$ be a principal $G$-bundle, i.e., $G$ acts freely and properly on $Q$. The diagonal action of $G$ on $Q \times Q$ is also free and proper, so we may form the quotient $(Q \times Q) / G$. Let $[q] \in Q / G$ denote the orbit of $q \in Q$ and $\left[q_{1}, q_{0}\right] \in(Q \times Q) / G$ denote the orbit of $\left(q_{1}, q_{0}\right) \in Q \times Q$. Then the gauge groupoid (or Atiyah groupoid) $(Q \times Q) / G \rightrightarrows Q / G$ of the principal bundle is defined by the structure maps

$$
\begin{aligned}
& \alpha\left(\left[q_{1}, q_{0}\right]\right)=\left[q_{0}\right], \quad \beta\left(\left[q_{1}, q_{0}\right]\right)=\left[q_{1}\right], \quad m\left(\left[q_{2}, q_{1}\right],\left[q_{1}, q_{0}\right]\right)=\left[q_{2}, q_{0}\right], \\
& \epsilon([q])=[q, q], \quad i\left(\left[q_{1}, q_{0}\right]\right)=\left[q_{0}, q_{1}\right] .
\end{aligned}
$$

Notice that $G \rightrightarrows \bullet$ is the special case where $Q=G$ acts on itself by multiplication, while $Q \times Q \rightrightarrows Q$ is the special case where $G=\{e\}$ acts trivially on $Q$.

Definition 4.6. A left action (or just action) of a Lie groupoid $G \rightrightarrows M$ on a fibered manifold $Q \rightarrow M$ is a smooth map $G_{\alpha} \times{ }_{\mu} Q \rightarrow Q,(g, q) \mapsto g q$, such that

(i) $\mu(g q)=\beta(g)$ for all $(g, q) \in G_{\alpha} \times{ }_{\mu} Q$,

(ii) $g(h q)=(g h) q$ for all $(g, h, q) \in G_{\alpha} \times{ }_{\beta} G_{\alpha} \times{ }_{\mu} Q$, and

(iii) $\epsilon(\mu(q)) q=q$ for all $q \in Q$.

The action is free if $g q=q$ implies $g=\epsilon(\mu(q))$, and it is proper if its graph,

$$
G_{\alpha} \times{ }_{\mu} Q \rightarrow Q \times Q, \quad(g, q) \mapsto(g q, q),
$$

is a proper map. A principal $G$-space is a fibered manifold endowed with a free and proper $G$-action.

Remark 4.7. As with group actions, it can be shown that if $G \rightrightarrows M$ acts freely and properly on $Q \rightarrow M$, then the quotient $Q / G$ consisting of $G$-orbits is a smooth manifold, and there is a smooth quotient map $Q \rightarrow Q / G$. We refer to Dufour and Zung [8, Chapter 7] for a more detailed discussion of this and other properties of groupoid actions. 
Example 4.8. The action of a Lie group $G$ on a manifold $Q$ is precisely the action of the Lie groupoid $G \rightrightarrows \bullet$ on the fibered manifold $Q \rightarrow \bullet$. If the action is free and proper, then the associated principal $G$-space corresponds to the principal $G$-bundle $Q \rightarrow Q / G$.

Example 4.9. For any smooth manifold $Q$, the pair groupoid $Q \times Q \rightrightarrows Q$ acts on $Q$ by $\left(q_{1}, q_{0}\right) q_{0}=q_{1}$. (In this case, we treat $Q$ as the fibered manifold $Q \rightarrow Q$, rather than $Q \rightarrow \bullet$.) Since any two points $q_{0}, q_{1}$ lie in the same orbit, it follows that $Q /(Q \times Q) \cong \bullet$, and the quotient map is simply $Q \rightarrow \bullet$.

Example 4.10. Let $G$ be a Lie group acting freely and properly on $Q$, so that $Q \rightarrow Q / G$ is a principal $G$-bundle. Then the gauge groupoid $(Q \times Q) / G$ acts on $Q \rightarrow Q / G$, in the sense of Definition 4.6, and is uniquely defined by the condition $\left[q_{1}, q_{0}\right] q_{0}=q_{1}$. (Notice that Example 4.9 is the special case where $G=\{e\}$ acts trivially on $Q$.) Again, we see that any two points $q_{0}, q_{1} \in Q$ lie in the same orbit, so $Q /((Q \times Q) / G) \cong \bullet$, and the quotient map is $Q \rightarrow \bullet$.

Example 4.11. For any Lie groupoid $G \rightrightarrows M$, the multiplication map $m$ is an action of $G$ on itself, treated as the fibered manifold $\beta: G \rightarrow M$. This action is free, since $g h=h$ implies $g=(g h) h^{-1}=h h^{-1}=\epsilon(\beta(h))$. Moreover, the action is proper: $(g, h) \mapsto(g h, h)$ is a diffeomorphism, having the inverse $(g, h) \mapsto\left(g h^{-1}, h\right)$, so in particular it is a proper map.

The orbit of each $h \in G$ is its $\alpha$-fiber $\alpha^{-1}(\{x\})$, where $x=\alpha(h)$. Identifying the fiber $\alpha^{-1}(\{x\})$ with the corresponding base point $x \in M$, it follows that $G / G \cong M$, and the quotient map is just $\alpha: G \rightarrow M$.

Example 4.12. If $G \rightrightarrows M$ acts on $Q \rightarrow M$, then it also acts on $V Q \rightarrow M$, considered as a fibered manifold. Specifically, we have the action

$$
G_{\alpha} \times_{\mu \circ \tau} V Q \rightarrow V Q, \quad(g, v) \mapsto g_{*} v,
$$

where $g_{*}$ denotes the pushforward of $q \mapsto g q$.

Lemma 4.13. Suppose $G \rightrightarrows M$ has a free, proper action on $Q \rightarrow M$. Then its diagonal action on $Q_{\mu} \times_{\mu} Q \rightarrow M$, given by $g\left(q_{1}, q_{0}\right)=\left(g q_{1}, g q_{0}\right)$, is also free and proper. Moreover, the quotient can be given a natural Lie group structure $\left(Q_{\mu} \times_{\mu} Q\right) / G \rightrightarrows Q / G$, and the quotient map $Q{ }_{\mu} \times{ }_{\mu} Q \rightarrow\left(Q{ }_{\mu} \times{ }_{\mu} Q\right) / G$ is a morphism of Lie groupoids over $Q \rightarrow Q / G$.

Proof. The fact that $\left(g,\left(q_{1}, q_{0}\right)\right) \mapsto\left(g q_{1}, g q_{0}\right)$ is a free and proper groupoid action follows immediately from the fact that, by assumption, $(g, q) \mapsto g q$ is. As stated in Remark 4.7, the freeness and properness of these actions imply that $Q / G$ and $\left(Q_{\mu} \times_{\mu} Q\right) / G$ are smooth manifolds, so it suffices to specify the groupoid structure maps for $\left(Q_{\mu} \times_{\mu} Q\right) / G \rightrightarrows Q / G$. These may be taken to be formally identical to those for the gauge groupoid in Example 4.5, i.e.,

$$
\begin{aligned}
& \alpha\left(\left[q_{1}, q_{0}\right]\right)=\left[q_{0}\right], \quad \beta\left(\left[q_{1}, q_{0}\right]\right)=\left[q_{1}\right], \quad m\left(\left[q_{2}, q_{1}\right],\left[q_{1}, q_{0}\right]\right)=\left[q_{2}, q_{0}\right], \\
& \epsilon([q])=[q, q], \quad i\left(\left[q_{1}, q_{0}\right]\right)=\left[q_{0}, q_{1}\right] .
\end{aligned}
$$

As with the gauge groupoid, it is simple to check directly that these satisfy the conditions of Definition 4.1, so this is a Lie groupoid. Finally, using $\widetilde{\alpha}, \widetilde{\beta}, \ldots$ to denote the structure maps on $Q{ }_{\mu} \times{ }_{\mu} Q \rightrightarrows Q$, we observe that

$$
\begin{aligned}
& \alpha\left(\left[q_{1}, q_{0}\right]\right)=\left[\widetilde{\alpha}\left(q_{1}, q_{0}\right)\right], \quad \beta\left(\left[q_{1}, q_{0}\right]\right)=\left[\widetilde{\beta}\left(q_{1}, q_{0}\right)\right], \\
& m\left(\left[q_{2}, q_{1}\right],\left[q_{1}, q_{0}\right]\right)=\left[\widetilde{m}\left(\left(q_{2}, q_{1}\right),\left(q_{1}, q_{0}\right)\right)\right], \\
& \epsilon([q])=[\widetilde{\epsilon}(q)], \quad i\left(\left[q_{1}, q_{0}\right]\right)=\left[\widetilde{\imath}\left(q_{1}, q_{0}\right)\right],
\end{aligned}
$$

so the quotient map preserves the structure maps and hence is a Lie groupoid morphism. 
Lemma 4.14. The action of a Lie groupoid $G \rightrightarrows M$ on $Q \rightarrow M$ is free (resp., proper) if and only if the induced action on $V Q \rightarrow M$ is free (resp., proper).

Proof. If $G$ acts freely on $Q$, then $g_{*} v=v$ implies $g(\tau(v))=\tau(v)$, so $g=\epsilon(\mu(\tau(v)))=$ $\epsilon((\mu \circ \tau)(v))$, and hence $G$ acts freely on $V Q$. Conversely, if $G$ acts freely on $V Q$, then $g q=q$ implies $g_{*} 0_{q}=0_{q}$ so $g=\epsilon\left((\mu \circ \tau)\left(0_{q}\right)\right)=\epsilon(\mu(q))$, and hence $G$ acts freely on $Q$.

The proof of properness essentially amounts to chasing compact sets around the following diagram:

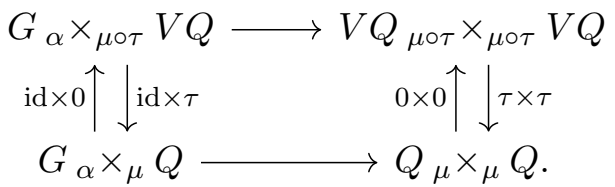

First, suppose $G$ acts properly on $Q$. If $K \subset V Q{ }_{\mu \circ \tau} \times_{\mu \circ \tau} V Q$ is compact, then we wish to show that the preimage,

$$
\left\{(g, v) \in G_{\alpha} \times_{\mu \circ \tau} V Q:\left(v, g_{*} v\right) \in K\right\},
$$

is also compact. Observe that $\left\{v \in V Q:\left(v, g_{*} v\right) \in K\right\}$ is compact by the continuity of $\left(v, g_{*} v\right) \mapsto v$, and $\left\{g \in G:\left(v, g_{*} v\right) \in K\right\}$ is compact by the continuity of $\left(v, g_{*} v\right) \mapsto(q, g q)$, with $q=\tau(v)$, the properness of $(g, q) \mapsto(q, g q)$, and the continuity of $(g, q) \mapsto g$. Hence, the preimage in question is also compact, so $G$ acts properly on $V Q$.

Conversely, suppose $G$ acts properly on $V Q$. If $K \subset Q_{\mu} \times{ }_{\mu} Q$ is compact, then so is $\left\{\left(0_{q}, g_{*} 0_{q}\right) \in\right.$ $\left.V Q_{\mu \circ \tau} \times_{\mu \circ \tau} V Q:(q, g q) \in K\right\}$, and by properness, so is $\left\{\left(g, 0_{q}\right) \in G_{\alpha} \times_{\mu \circ \tau} V Q:(q, g q) \in K\right\}$. Finally, the preimage,

$$
\left\{(g, q) \in G_{\alpha} \times{ }_{\mu} Q:(g, q) \in K\right\},
$$

is compact by the continuity of $\left(g, 0_{q}\right) \mapsto(g, q)$, so $G$ acts properly on $Q$.

\subsection{Lie algebroid of a Lie groupoid}

Before discussing reduction by an arbitrary free and proper groupoid action, we first consider the important special case where a groupoid acts on itself by left multiplication. (This can be thought of as the "groupoid version" of Euler-Poincaré reduction, which is the special case of Lagrange-Poincaré reduction where $Q=G$ is a Lie group.)

Recall from Example 4.11 that a Lie groupoid $G \rightrightarrows M$ acts freely and properly on itself (as the fibered manifold $\beta: G \rightarrow M$ ) by left multiplication. Lemma 4.14 implies that this induces a free and proper action of $G$ on the $\beta$-vertical bundle $V^{\beta} G \rightarrow M$. (Since $G$ can be seen as a fibered manifold in two different ways, $\alpha: G \rightarrow M$ and $\beta: G \rightarrow M$, we denote the corresponding vertical bundles by $V^{\alpha} G$ and $V^{\beta} G$ to avoid any possible confusion.) Since the orbit of $v \in V_{g}^{\beta} G$ is uniquely determined by its representative at the identity section, $\left(g^{-1}\right)_{*} v \in V_{\epsilon(\alpha(g))}^{\beta} G$, we can identify the quotient $V^{\beta} G / G$ with the vector bundle $A G=V_{\epsilon(M)}^{\beta} G$ over $M$.

This vector bundle $A G \rightarrow M$ is in fact a Lie algebroid, called the Lie algebroid of $G$. The anchor map is given by the restriction of $\alpha_{*}: T G \rightarrow T M$ to $A G$. Furthermore, the identification of $A G$ with $V^{\beta} G / G$ implies that sections $X \in \Gamma(A G)$ correspond to $G$-invariant, $\beta$-vertical vector fields $\overleftarrow{X} \in \mathfrak{X}_{\beta}(G)$, with $\overleftarrow{X}(g)=g_{*} X(\alpha(g))$. The bracket $[X, Y]$ of $X, Y \in \Gamma(A G)$ is then defined so that $\overleftarrow{X, Y]}=[\overleftarrow{X}, \overleftarrow{Y}]$, where the bracket on the right-hand side of this expression is just the Jacobi-Lie bracket of vector fields on $G$. (See Mackenzie [14].) 
Example 4.15. Let $G$ be a Lie group, so that $G \rightrightarrows \bullet$ is a Lie groupoid. Since $\beta$ is trivial, we have $V^{\beta} G=T G$, and hence $A G=T_{e} G=\mathfrak{g} \rightarrow \bullet$, where $\mathfrak{g}$ is the Lie algebra of $G$ and $e=\epsilon(\bullet) \in G$ is the identity element of $G$.

Example 4.16. For the pair groupoid $Q \times Q \rightrightarrows Q$, we have $V^{\beta}(Q \times Q)=T Q \times Q$, and hence $A(Q \times Q)=T Q_{\tau} \times Q \cong T Q \rightarrow Q$.

More generally, if we consider the groupoid $Q_{\mu} \times{ }_{\mu} Q \rightrightarrows Q$ for a fibered manifold $Q \rightarrow M$, then $V^{\beta}\left(Q_{\mu} \times{ }_{\mu} Q\right)=V Q_{\mu \circ \tau} \times_{\mu} Q$, and hence $A\left(Q_{\mu} \times_{\mu} Q\right)=V Q_{\tau} \times Q \cong V Q \rightarrow Q$.

Example 4.17. For the gauge groupoid $(Q \times Q) / G \rightrightarrows Q / G$ of a principal bundle $Q \rightarrow Q / G$, we have $V^{\beta}((Q \times Q) / G)=(T Q \times Q) / G$, and hence $A((Q \times Q) / G)=\left(T Q_{\tau} \times Q\right) / G \cong T Q / G \rightarrow Q / G$. This is called the gauge algebroid (or Atiyah algebroid) of the principal bundle.

More generally, considering the groupoid $\left(Q_{\mu} \times_{\mu} Q\right) / G \rightrightarrows G$ of a principal $G$-space, we have $V^{\beta}\left(\left(Q_{\mu} \times{ }_{\mu} Q\right) / G\right)=\left(V Q_{\mu \circ \tau} \times{ }_{\mu} Q\right) / G$, and hence $A\left(\left(Q_{\mu} \times{ }_{\mu} Q\right) / G\right)=\left(V Q_{\tau} \times Q\right) / G \cong V Q / G \rightarrow$ $Q / G$.

Remark 4.18. The relationship between a groupoid $G$ and its algebroid $A G$ has an interesting application to the discretization of Lagrangian mechanics, which can be used to develop structurepreserving numerical integrators. In this approach, pioneered by Weinstein [30] (see also Marrero et al. [15, 16], Stern [29]), one replaces the Lagrangian $L: A G \rightarrow \mathbb{R}$ by a discrete Lagrangian $L_{h}: G \rightarrow \mathbb{R}$, replaces $A G$-paths by sequences of composable arrows in $G$, and uses a variational principle to derive discrete equations of motion. In particular, using $G=Q \times Q \rightrightarrows Q$ to discretize $A G=T Q \rightarrow Q$ gives the framework of variational integrators (cf. Moser and Veselov [27], Marsden and West [22]).

\subsection{Reduction by a groupoid action}

Recall from Lemma 4.14 that if $G \rightrightarrows M$ acts freely and properly on $Q \rightarrow M$, then it also acts freely and properly on $V Q \rightarrow M$. In other words, $V Q$ is also a principal $G$-space, equipped with a quotient map $V Q \rightarrow V Q / G$. We have seen that $V Q$ is also a Lie algebroid, and moreover, in Example 4.16, that it is the Lie algebroid of the Lie groupoid $Q_{\mu} \times_{\mu} Q \rightrightarrows Q$. Similarly, from Example 4.17, we have that $V Q / G$ is the Lie algebroid of the Lie groupoid $\left(Q_{\mu} \times{ }_{\mu} Q\right) / G \rightrightarrows Q / G$.

Therefore, in order to perform reduction using Theorem 3.19, it suffices to show that the quotient map $V Q \rightarrow V Q / G$ is in fact a Lie algebroid morphism.

Lemma 4.19. Let $G \rightrightarrows M$ be a Lie groupoid and $Q \rightarrow M$ a principal $G$-space. Then the quotient map $V Q \rightarrow V Q / G$ is a Lie algebroid morphism covering $Q \rightarrow Q / G$.

Proof. We can use a result stated in Mackenzie [14, Proposition 4.3.4], which says that a morphism of Lie groupoids $G \rightarrow G^{\prime}$ induces a corresponding morphism of Lie algebroids $A G \rightarrow A G^{\prime}$. This defines the so-called Lie functor between the categories of Lie groupoids and Lie algebroids, taking objects $G \mapsto A G$ and morphisms $\left(G \rightarrow G^{\prime}\right) \mapsto\left(A G \rightarrow A G^{\prime}\right)$.

Now, we have already proved in Lemma 4.13 that the quotient map $Q_{\mu} \times{ }_{\mu} Q \rightarrow\left(Q_{\mu} \times{ }_{\mu} Q\right) / G$ is a morphism of Lie groupoids, so applying the Lie functor to this morphism proves the result.

Theorem 4.20. Let $G \rightrightarrows M$ be a Lie groupoid and $Q \rightarrow M$ a principal $G$-space. Suppose the Lagrangian $L: V Q \rightarrow \mathbb{R}$ is $G$-invariant, i.e., that it factors through the quotient morphism $\Phi: V Q \rightarrow V Q / G$ as $L=\ell \circ \Phi$, where $\ell: V Q / G \rightarrow \mathbb{R}$ is called the reduced Lagrangian. Then $a \in \mathcal{P}_{V}(Q)$ is a solution path for $L$ if and only if $\Phi \circ a \in \mathcal{P}_{\rho}(V Q / G)$ is a solution path for $\ell$.

Proof. Apply Theorem 3.19 to the (fiberwise-surjective) Lie algebroid morphism defined in Lemma 4.19. 
Example 4.21. When $G \rightrightarrows \bullet$ is a Lie group acting freely and properly on $Q \rightarrow \bullet$, Theorem 4.20 corresponds to ordinary Lagrangian reduction from $T Q$ to $T Q / G$, yielding the Lagrange-Poincaré equations of Section 3.4. In the special case where $Q=G$ acts on itself by multiplication, this gives Euler-Poincaré reduction from $T G$ to $T G / G \cong \mathfrak{g}$.

Example 4.22. Suppose $G \rightrightarrows M$ is a Lie groupoid acting on itself by multiplication, so that the quotient morphism is $\Phi: V^{\beta} G \rightarrow V^{\beta} G / G=A G$. If $L: V^{\beta} G \rightarrow \mathbb{R}$ and $\ell: A G \rightarrow \mathbb{R}$ are Lagrangians satisfying $L=\ell \circ \Phi$, then Theorem 4.20 implies that the vertical Euler-Lagrange equations (Section 2) on $V^{\beta} G$ reduce to the Euler-Lagrange-Poincaré equations (Section 3) for the Lie algebroid $A G$. (This special case appears in Weinstein [30, Theorem 5.3].) The even more special case where $G \rightrightarrows \bullet$ is a Lie group again gives Euler-Poincaré reduction on the Lie algebra $\mathfrak{g}$.

\section{The Hamilton-Pontryagin principle and reduction}

In this section, we extend the foregoing theory to the Hamilton-Pontryagin variational principle introduced by Yoshimura and Marsden [33] as a generalization of Hamilton's variational principle. This principle is especially useful for the study of "implicit Lagrangian systems" that arise in mechanical and control systems with nonholonomic or Dirac constraints. (See also Yoshimura and Marsden [32] for the non-variational approach to such systems, as well as Yoshimura and Marsden [34] for the associated reduction theory.)

We begin, in Section 5.1, with a brief review of the Hamilton-Pontryagin principle for ordinary manifolds. We then generalize it, in Section 5.2, to fibered manifolds and their (co)vertical bundles, as we did for Hamilton's principle in Section 2. In Section 5.3, we generalize the Hamilton-Pontryagin principle even further to mechanics on Lie algebroids and their duals, as was done for Hamilton's principle in Section 3. Finally, in Section 5.4, we discuss reduction of the Hamilton-Pontryagin principle by Lie algebroid morphisms, as in the Weinstein-Martínez reduction theorem (Theorem 3.19), and apply this to the special case of groupoid symmetries for a fibered manifold, as in Theorem 4.20 .

\subsection{Hamilton-Pontryagin principle for ordinary manifolds}

We begin with a quick review of the Hamilton-Pontryagin principle for ordinary (non-fibered) manifolds, as introduced in Yoshimura and Marsden [33].

Let $L: T Q \rightarrow \mathbb{R}$ be a Lagrangian. The Hamilton-Pontryagin action is the functional $S: \mathcal{P}\left(T Q \oplus T^{*} Q\right) \rightarrow \mathbb{R}$ defined, in fiber coordinates, by

$$
S(q, v, p)=\int_{0}^{1}(L(q(t), v(t))+\langle p(t), \dot{q}(t)-v(t)\rangle) \mathrm{d} t .
$$

Here, $(q, v, p)$ is an arbitrary path in the Pontryagin bundle $T Q \oplus T^{*} Q$. We emphasize that no restrictions are placed on this path - in particular, the second-order curve condition $\dot{q}=v$ is not a priori required.

The path $(q, v, p)$ satisfies the Hamilton-Pontryagin principle if $\mathrm{d} S(\delta q, \delta v, \delta p)=0$ for all variations $(\delta q, \delta v, \delta p) \in T_{(q, v, p)} \mathcal{P}\left(T Q \oplus T^{*} Q\right)$ such that $\delta q(0)=0$ and $\delta q(1)=0$. (That is, the endpoints of $q$ are fixed, while the endpoints of $v$ and $p$ are unrestricted.) In local coordinates, we have

$$
\begin{aligned}
\mathrm{d} S(\delta q, \delta v, \delta p) & =\int_{0}^{1}\left(\frac{\partial L}{\partial q^{i}}(q, v) \delta q^{i}+\frac{\partial L}{\partial v^{i}}(q, v) \delta v^{i}+p_{i}\left(\delta \dot{q}^{i}-\delta v^{i}\right)+\delta p_{i}\left(\dot{q}^{i}-v^{i}\right)\right) \mathrm{d} t \\
& =\int_{0}^{1}\left[\left(\frac{\partial L}{\partial q^{i}}(q, v)-\dot{p}_{i}\right) \delta q^{i}+\left(\frac{\partial L}{\partial v^{i}}(q, v)-p_{i}\right) \delta v^{i}+\delta p_{i}\left(\dot{q}^{i}-v^{i}\right)\right] \mathrm{d} t .
\end{aligned}
$$


Hence, this vanishes when $(q, v, p)$ satisfies the differential-algebraic equations

$$
\frac{\partial L}{\partial q^{i}}(q, v)-\dot{p}_{i}=0, \quad \frac{\partial L}{\partial v^{i}}(q, v)-p_{i}=0, \quad \dot{q}^{i}-v^{i}=0,
$$

which Yoshimura and Marsden [33] call the implicit Euler-Lagrange equations. The three systems of equations correspond, respectively, to the Euler-Lagrange equations, the Legendre transform, and the second-order curve condition. (Note that the conjugate momentum $p$ acts like a "Lagrange multiplier" enforcing the second-order curve condition $\dot{q}=v$.)

In this sense, the Hamilton-Pontryagin approach generalizes and unifies the symplectic and variational approaches to Lagrangian mechanics.

\subsection{Hamilton-Pontryagin for fibered manifolds}

Suppose, more generally, that $L: V Q \rightarrow \mathbb{R}$ is a Lagrangian on the vertical bundle of a fibered manifold $Q \rightarrow M$. Recall that $V Q$ and $V^{*} Q$ can both be viewed as fibered manifolds over $M$, and thus so can $V Q \oplus V^{*} Q$, which we call the vertical Pontryagin bundle. It follows that we may define a Banach manifold of vertical paths $\mathcal{P}_{V}\left(V Q \oplus V^{*} Q\right)$ and its bundle of vertical variations $V \mathcal{P}_{V}\left(V Q \oplus V^{*} Q\right)$.

Definition 5.1. Given a Lagrangian $L: V Q \rightarrow \mathbb{R}$, the Hamilton-Pontryagin action $S: \mathcal{P}_{V}(V Q \oplus$ $\left.V^{*} Q\right) \rightarrow \mathbb{R}$ is defined, in fiber coordinates, by

$$
S(q, v, p)=\int_{0}^{1}(L(q(t), v(t))+\langle p(t), \dot{q}(t)-v(t)\rangle) \mathrm{d} t .
$$

A vertical path $(q, v, p) \in \mathcal{P}_{V}\left(V Q \oplus V^{*} Q\right)$ is said to satisfy the Hamilton-Pontryagin principle if $\mathrm{d} S(\delta q, \delta v, \delta p)=0$ for all vertical variations $(\delta q, \delta v, \delta p) \in V_{(q, v, p)} \mathcal{P}_{V}\left(V Q \oplus V^{*} Q\right)$ such that $\delta q(0)=0$ and $\delta q(1)=0$.

Theorem 5.2. A vertical path $(q, v, p) \in \mathcal{P}_{V}\left(V Q \oplus V^{*} Q\right)$ satisfies the Hamilton-Pontryagin principle if and only if, in fiber-adapted local coordinates $q=\left(x^{\sigma}, y^{i}\right)$, it satisfies the implicit vertical Euler-Lagrange equations,

$$
\dot{x}^{\sigma}=0, \quad \dot{p}_{i}=\frac{\partial L}{\partial y^{i}}(q, v), \quad p_{i}=\frac{\partial L}{\partial v^{i}}(q, v), \quad \dot{y}^{i}=v^{i} .
$$

Proof. The equations $\dot{x}^{\sigma}=0$ are simply the vertical path condition. Given a vertical variation $(\delta q, \delta v, \delta p) \in V_{(q, v, p)} \mathcal{P}_{V}\left(V Q \oplus V^{*} Q\right)$ satisfying $\delta q(0)=0$ and $\delta q(1)=0$,

$$
\begin{aligned}
\mathrm{d} S(\delta q, \delta v, \delta p) & =\int_{0}^{1}\left(\frac{\partial L}{\partial y^{i}}(q, v) \delta y^{i}+\frac{\partial L}{\partial v^{i}}(q, v) \delta v^{i}+p_{i}\left(\delta \dot{y}^{i}-\delta v^{i}\right)+\delta p_{i}\left(\dot{y}^{i}-v^{i}\right)\right) \mathrm{d} t \\
& =\int_{0}^{1}\left[\left(\frac{\partial L}{\partial y^{i}}(q, v)-\dot{p}_{i}\right) \delta y^{i}+\left(\frac{\partial L}{\partial v^{i}}(q, v)-p_{i}\right) \delta v^{i}+\delta p_{i}\left(\dot{y}^{i}-v^{i}\right)\right] \mathrm{d} t .
\end{aligned}
$$

This vanishes for arbitrary $(\delta q, \delta v, \delta p)$ if and only if each of the components in the integrand vanishes, which completes the proof.

\subsection{Hamilton-Pontryagin for arbitrary Lie algebroids}

We next generalize the Hamilton-Pontryagin principle to a Lagrangian $L: A \rightarrow \mathbb{R}$, where $A \rightarrow Q$ is an arbitrary Lie algebroid. The previous subsections will then correspond to the special cases $A=T Q$ and $A=V Q$, respectively.

One might expect that the appropriate generalization of paths in $T Q \oplus T^{*} Q$ or $V Q \oplus V^{*} Q$ would be paths in $A \oplus A^{*}$. However, these generally do not contain sufficient information to recover the $A$-path condition (the generalization of the second-order curve condition). Instead, we consider an alternative class of paths that we call $\left(A, A^{*}\right)$-paths. 
Definition 5.3. An $\left(A, A^{*}\right)$-path consists of the following components:

(i) an $A$-path $a \in \mathcal{P}_{\rho}(A)$ over some base path $q \in \mathcal{P}(Q)$;

(ii) a path $v \in \mathcal{P}(A)$, not necessarily an $A$-path, over $q$;

(iii) a path $p \in \mathcal{P}\left(A^{*}\right)$ over $q$.

We denote this by $(a, v, p) \in \mathcal{P}\left(A, A^{*}\right)$.

Example 5.4. Any path $(q, v, p) \in \mathcal{P}\left(T Q \oplus T^{*} Q\right)$ can be identified with the $\left(T Q, T^{*} Q\right)$-path $(\dot{q}, v, p) \in \mathcal{P}\left(T Q, T^{*} Q\right)$. More generally, $(q, v, p) \in \mathcal{P}_{V}\left(V Q \oplus V^{*} Q\right)$ can be identified with $(\dot{q}, v, p) \in \mathcal{P}\left(V Q, V^{*} Q\right)$. Thus, $\mathcal{P}\left(V Q, V^{*} Q\right) \cong \mathcal{P}_{V}\left(V Q \oplus V^{*} Q\right)$.

In this special case, the base path has a unique $A$-path prolongation, so it suffices to consider paths in $A \oplus A^{*}$ - but this is not the case in general.

Example 5.5. Let $\mathfrak{g}$ be a Lie algebra. Since all paths in $\mathfrak{g} \rightarrow \bullet$ are $\mathfrak{g}$-paths, it follows that a $\left(\mathfrak{g}, \mathfrak{g}^{*}\right)$ path $(a, v, p) \in \mathcal{P}\left(\mathfrak{g}, \mathfrak{g}^{*}\right)$ consists of two (generally distinct) paths $a, v \in \mathcal{P}(\mathfrak{g})$ and a path $p \in \mathcal{P}\left(\mathfrak{g}^{*}\right)$. Thus, $\mathcal{P}\left(\mathfrak{g}, \mathfrak{g}^{*}\right) \cong \mathcal{P}\left(\mathfrak{g} \oplus \mathfrak{g} \oplus \mathfrak{g}^{*}\right)$.

Definition 5.6. An admissible variation of $(a, v, p) \in \mathcal{P}\left(A, A^{*}\right)$ consists of an admissible variation $X_{b, a} \in \mathcal{F}_{a}(A)$ of the $A$-path $a$, together with arbitrary variations $\delta v \in T_{v} \mathcal{P}(A)$ and $\delta p \in T_{p} \mathcal{P}\left(A^{*}\right)$, such that all agree on the horizontal component $\delta q=\rho(b) \in \mathcal{P}_{q}(Q)$. That is, if $\tau: A \rightarrow Q$ and $\pi: A^{*} \rightarrow Q$ are the bundle projections, we require $\tau_{*}(v)=\pi_{*}(p)=\rho(b)$. Following Remark 3.10, we denote this subbundle of admissible variations by $\mathcal{F}\left(A, A^{*}\right) \subset T \mathcal{P}\left(A, A^{*}\right)$.

Remark 5.7. Given a $T Q$-connection $\nabla$, the admissible variation $\left(X_{b, a}, \delta v, \delta p\right) \in \mathcal{F}_{(a, v, p)}\left(A, A^{*}\right)$ has components $X_{b, a}^{\mathrm{ver}}=\bar{\nabla}_{a} b$ and $X_{b, a}^{\text {hor }}=\delta v^{\text {hor }}=\delta p^{\text {hor }}=\rho(b)$, while $\delta v^{\text {ver }}$ and $\delta p^{\text {ver }}$ are arbitrary paths in $A$ and $A^{*}$, respectively.

Example 5.8. Continuing Example 5.5, let us consider the case where $\mathfrak{g}$ is a Lie algebra. Given a $\left(\mathfrak{g}, \mathfrak{g}^{*}\right)$ path $(a, v, p)$, we identify $a$ with its time-dependent section $\xi(t)=\xi(t, \bullet)=a(t)$. Then an admissible variation of $(a, v, p)$ has the form $(\dot{\xi}+[\xi, \eta], \delta v, \delta p)$, where $\eta, \delta v$, and $\delta p$ are arbitrary paths in $\mathfrak{g}$.

Equivalently, assuming $\mathfrak{g}$ is the Lie algebra of a Lie group $G$, let $g \in \mathcal{P}(G)$ be a path integrating $\xi$, i.e., $g(t)=g(0) \exp (t \xi)$, so that $\xi=\left(g^{-1}\right)_{*} \dot{g}$. It follows that arbitrary variations $(\delta g, \delta v, \delta p) \in T_{(g, v, p)} \in \mathcal{P}\left(G \times \mathfrak{g} \times \mathfrak{g}^{*}\right)$ correspond precisely to admissible variations $(\dot{\xi}+[\xi, \eta], \delta v, \delta p)$ of $(\xi, v, p) \in \mathcal{P}\left(\mathfrak{g}, \mathfrak{g}^{*}\right)$, where $\eta=\left(g^{-1}\right)_{*} \delta g$. This special case corresponds to the approach of Yoshimura and Marsden [34] and Bou-Rabee and Marsden [2] for Hamilton-Pontryagin mechanics on Lie algebras, where one considers paths in $\mathcal{P}\left(G \times \mathfrak{g} \times \mathfrak{g}^{*}\right)$ and then left-reduces by $G$.

Definition 5.9. Given a Lagrangian $L: A \rightarrow \mathbb{R}$, the Hamilton-Pontryagin action $S: \mathcal{P}\left(A, A^{*}\right) \rightarrow$ $\mathbb{R}$ is defined by

$$
S(a, v, p)=\int_{0}^{1}(L(v(t))+\langle p(t), a(t)-v(t)\rangle) \mathrm{d} t,
$$

and $(a, v, p) \in \mathcal{P}\left(A, A^{*}\right)$ is said to satisfy the Hamilton-Pontryagin principle if $\mathrm{d} S\left(X_{b, a}, \delta v, \delta p\right)=0$ for all admissible variations $\left(X_{b, a}, \delta v, \delta p\right) \in \mathcal{F}_{(a, v, p)}\left(A, A^{*}\right)$.

Theorem 5.10. An $\left(A, A^{*}\right)$-path $(a, v, p) \in \mathcal{P}\left(A, A^{*}\right)$ satisfies the Hamilton-Pontryagin principle if and only if, given a TQ-connection $\nabla$ on $A$, it satisfies the differential-algebraic equations,

$$
\rho^{*} \mathrm{~d} L^{\mathrm{hor}}(v)+\bar{\nabla}_{a}^{*} p=0, \quad \mathrm{~d} L^{\mathrm{ver}}(v)-p=0, \quad a-v=0 .
$$


Proof. Given $\left(X_{b, a}, \delta v, \delta p\right) \in \mathcal{F}_{(a, v, p)}\left(A, A^{*}\right)$, we compute

$$
\begin{aligned}
\mathrm{d} S\left(X_{b, a}, \delta v, \delta p\right)= & \int_{0}^{1}\left(\left\langle\mathrm{~d} L^{\text {hor }}(v), \rho(b)\right\rangle+\left\langle\mathrm{d} L^{\text {ver }}(v), \delta v^{\text {ver }}\right\rangle\right. \\
& \left.\quad+\left\langle p, \bar{\nabla}_{a} b-\delta v^{\text {ver }}\right\rangle+\left\langle\delta p^{\text {ver }}, a-v\right\rangle\right) \mathrm{d} t \\
= & \int_{0}^{1}\left(\left\langle\rho^{*} \mathrm{~d} L^{\text {hor }}(v)+\bar{\nabla}_{a}^{*} p, b\right\rangle+\left\langle\mathrm{d} L^{\text {ver }}(v)-p, \delta v^{\text {ver }}\right\rangle+\left\langle\delta p^{\text {ver }}, a-v\right\rangle\right) \mathrm{d} t .
\end{aligned}
$$

The Hamilton-Pontryagin principle is satisfied if and only if each term in the integrand vanishes, and since $b, \delta v^{\text {ver }}$, and $\delta p^{\text {ver }}$ are arbitrary, the result follows.

We call the differential-algebraic equations (5.2) the implicit Euler-Lagrange-Poincaré equations. As we did in Theorem 3.14 we can give an equivalent expression for (5.2) in local coordinates.

Theorem 5.11. Let $q^{i}$ be local coordinates for $Q,\left\{e_{I}\right\}$ be a local basis of sections of $A,\left\{e^{I}\right\}$ be the dual basis of local sections of $A^{*}, \nabla$ be the locally trivial TQ-connection, and $\rho_{I}^{i}$ and $C_{I J}^{K}$ be the local-coordinate representations of $\rho$ and $[\cdot, \cdot]$. Let $(a, v, p) \in \mathcal{P}\left(A \oplus A \oplus A^{*}\right)$ have the localcoordinate representations $a(t)=\xi^{I}(t) e_{I}(q(t)), v(t)=v^{I}(t) e_{I}(q(t))$, and $p(t)=p_{I}(t) e^{I}(q(t))$. Then $(a, v, p) \in \mathcal{P}\left(A, A^{*}\right)$ if and only if $\dot{q}^{i}=\rho_{I}^{i} \xi^{I}$, and $(a, v, p)$ satisfies the implicit EulerLagrange-Poincaré equations (5.2) if and only if

$$
\rho_{I}^{i} \frac{\partial L}{\partial q^{i}}-C_{I J}^{K} \xi^{J} p_{K}-\dot{p}_{I}=0, \quad \frac{\partial L}{\partial \xi^{I}}-p_{I}=0, \quad \xi^{I}-v^{I}=0 .
$$

Proof. The proof is a straightforward computation, following Theorem 3.14.

\subsection{Reduction by groupoid symmetries}

Finally, we consider the reduction of Hamilton-Pontryagin mechanics by a Lie algebroid morphism $\Phi: A \rightarrow A^{\prime}$, as in Theorem 3.19. Here, though, we will require the slightly stronger assumption that $\Phi$ be a fiberwise isomorphism. (This was actually assumed in the original Lie algebroid reduction theorem of Weinstein [30], although Martínez [25] showed that it could be relaxed.) This stronger assumption is needed since $\Phi^{*}: A^{*} \rightarrow A^{*}$ points in the "wrong direction" for reduction from $\left(A, A^{*}\right)$ to $\left(A^{\prime}, A^{\prime *}\right)$, so we need fiberwise invertibility to map $A^{*} \rightarrow A^{\prime *}$.

Theorem 5.12. Let $\Phi: A \rightarrow A^{\prime}$ be a morphism of Lie algebroids, and suppose $L: A \rightarrow \mathbb{R}$ and $L^{\prime}: A^{\prime} \rightarrow \mathbb{R}$ are Lagrangians such that $L=L^{\prime} \circ \Phi$. If $\Phi$ is a fiberwise isomorphism, then $(a, v, p) \in \mathcal{P}\left(A, A^{*}\right)$ satisfies the Hamilton-Pontryagin principle for $L$ if and only if $\left(a^{\prime}, v^{\prime}, p^{\prime}\right) \in$ $\mathcal{P}\left(A^{\prime}, A^{\prime *}\right)$ satisfies the Hamilton-Pontryagin principle for $L^{\prime}$, where $a^{\prime}=\Phi \circ a, v^{\prime}=\Phi \circ v$, and $p^{\prime}=\left(\Phi^{*}\right)^{-1} \circ p$.

Proof. This can be shown directly from the variational principle - observing that admissible variations in $\mathcal{F}_{(a, v, p)}\left(A, A^{*}\right)$ map to those in $\mathcal{F}_{\left(a^{\prime}, v^{\prime}, p^{\prime}\right)}\left(A^{\prime}, A^{\prime *}\right)$, and vice versa - but we give an equivalent proof using the implicit Euler-Lagrange-Poincaré equations together with the Weinstein-Martínez reduction theorem (Theorem 3.19).

First, since $\Phi$ is a fiberwise isomorphism, we have $a=v$ if and only if $a^{\prime}=v^{\prime}$. Moreover, since $L=L^{\prime} \circ \Phi$, the following diagram commutes:

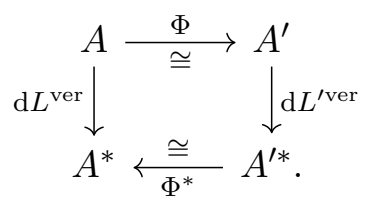


It follows from this that $p=\mathrm{d} L^{\mathrm{ver}}(v)$ if and only if $p^{\prime}=\mathrm{d} L^{\prime \text { ver }}\left(v^{\prime}\right)$. Finally, substituting these expressions for $v$ and $p$ into the first equation in (5.2), we have

$$
\rho^{*} \mathrm{~d} L^{\mathrm{hor}}(a)+\bar{\nabla}_{a}^{*} \mathrm{~d} L^{\mathrm{ver}}(a)=0, \quad \rho^{\prime *} \mathrm{~d} L^{\prime \text { hor }}\left(a^{\prime}\right)+\bar{\nabla}_{a^{\prime}}^{\prime *} \mathrm{~d} L^{\prime \text { ver }}\left(a^{\prime}\right)=0 .
$$

But these are just the Euler-Lagrange-Poincaré equations (3.1) for $L$ and $L^{\prime}$, respectively. So Theorem 3.19 implies that one holds if and only if the other does.

Fortunately, the fiberwise isomorphism assumption is still sufficient to perform reduction when $A=V Q \rightarrow Q$ and $A^{\prime}=V Q / G \rightarrow Q / G$, since the quotient map for the groupoid action in Lemma 4.19 is a fiberwise isomorphism. (Indeed, Higgins and Mackenzie [11] refer to Lie algebroid morphisms with this property as action morphisms.) Intuitively, this is because the quotient is taken both on the total space and on the base, so the dimension of the fibers remains the same.

Theorem 5.13. Let $G \rightrightarrows M$ be a Lie groupoid and $Q \rightarrow M$ a principal $G$-space. Suppose the Lagrangian $L: V Q \rightarrow \mathbb{R}$ is G-invariant, i.e., that it factors through the quotient morphism $\Phi: V Q \rightarrow V Q / G$ as $L=\ell \circ \Phi$, where $\ell: V Q / G \rightarrow \mathbb{R}$ is called the reduced Lagrangian. Then $(a, v, p) \in \mathcal{P}\left(V Q, V^{*} Q\right)$ satisfies the Hamilton-Pontryagin principle for $L$ if and only if $\left(a^{\prime}, v^{\prime}, p^{\prime}\right) \in \mathcal{P}\left(V Q / G, V^{*} Q / G\right)$ satisfies the Hamilton-Pontryagin principle for $\ell$, where $a^{\prime}=\Phi \circ a$, $v^{\prime}=\Phi \circ v$, and $p^{\prime}=\left(\Phi^{*}\right)^{-1} \circ p$.

Proof. Apply Theorem 5.12 to the quotient morphism $\Phi$, which is a fiberwise-isomorphic Lie algebroid morphism from $V Q$ to $V Q / G$.

Example 5.14. As in Example 4.21, when $G \rightrightarrows \bullet$ is a Lie group acting freely and properly on $Q \rightarrow \bullet$, this corresponds to the case of ordinary Lagrangian reduction for the HamiltonPontryagin principle. In the special case where $Q=G$ acts on itself by multiplication, this gives Euler-Poincaré-type reduction for the Hamilton-Pontryagin principle, as in Yoshimura and Marsden [34], Bou-Rabee and Marsden [2].

Example 5.15. As in Example 4.22, suppose $G \rightrightarrows M$ is a Lie groupoid acting on itself by multiplication, so that the quotient morphism is $\Phi: V^{\beta} G \rightarrow V^{\beta} G / G=A G$. If $L: V^{\beta} G \rightarrow \mathbb{R}$ and $\ell: A G \rightarrow \mathbb{R}$ are Lagrangians satisfying $L=\ell \circ \Phi$, then Theorem 5.13 implies that the implicit vertical Euler-Lagrange equations (5.1) on $V^{\beta} G$ reduce to the implicit Euler-Lagrange-Poincaré equations (5.2) on $A G$. The even more special case where $G \rightrightarrows \bullet$ is a Lie group again gives Hamilton-Pontryagin reduction from $G$ to $\mathfrak{g}$, as in Yoshimura and Marsden [34], Bou-Rabee and Marsden [2].

\section{Acknowledgments and disclosures}

The authors wish to thank Rui Loja Fernandes for his helpful feedback on this work. This paper also benefited substantially from the suggestions of the anonymous referees, to whom we wish to express our sincere gratitude. This research was supported in part by grants from the Simons Foundation (award 279968 to Ari Stern) and from the National Science Foundation (award DMS 1363250 to Xiang Tang). The authors declare that they have no conflict of interest.

\section{References}

[1] Arnold V.I., Kozlov V.V., Neishtadt A.I., Dynamical systems. III, Encyclopaedia of Mathematical Sciences, Vol. 3, Springer-Verlag, Berlin, 1988.

[2] Bou-Rabee N., Marsden J.E., Hamilton-Pontryagin integrators on Lie groups. I. Introduction and structurepreserving properties, Found. Comput. Math. 9 (2009), 197-219. 
[3] Cendra H., Marsden J.E., Ratiu T.S., Lagrangian reduction by stages, Mem. Amer. Math. Soc. 152 (2001), $\mathrm{x}+108$ pages.

[4] Cortés J., de León M., Marrero J.C., Martín de Diego D., Martínez E., A survey of Lagrangian mechanics and control on Lie algebroids and groupoids, Int. J. Geom. Methods Mod. Phys. 3 (2006), 509-558, math-ph/0511009.

[5] Cortés J., Martínez E., Mechanical control systems on Lie algebroids, IMA J. Math. Control Inform. 21 (2004), 457-492, math.OC/0402437.

[6] Crainic M., Fernandes R.L., Integrability of Lie brackets, Ann. of Math. 157 (2003), 575-620, math.DG/0105033.

[7] de León M., Marrero J.C., Martínez E., Lagrangian submanifolds and dynamics on Lie algebroids, J. Phys. A: Math. Gen. 38 (2005), R241-R308, math.DG/0407528.

[8] Dufour J.-P., Zung N.T., Poisson structures and their normal forms, Progress in Mathematics, Vol. 242, Birkhäuser Verlag, Basel, 2005.

[9] Grabowska K., Grabowski J., Variational calculus with constraints on general algebroids, J. Phys. A: Math. Theor. 41 (2008), 175204, 25 pages, arXiv:0712.2766.

[10] Grabowska K., Urbański P., Grabowski J., Geometrical mechanics on algebroids, Int. J. Geom. Methods Mod. Phys. 3 (2006), 559-575, math-ph/0509063.

[11] Higgins P.J., Mackenzie K.C.H., Duality for base-changing morphisms of vector bundles, modules, Lie algebroids and Poisson structures, Math. Proc. Cambridge Philos. Soc. 114 (1993), 471-488.

[12] Iglesias D., Marrero J.C., Martín de Diego D., Martínez E., Discrete nonholonomic Lagrangian systems on Lie groupoids, J. Nonlinear Sci. 18 (2008), 221-276, arXiv:0704.1543.

[13] Iglesias D., Marrero J.C., Martín de Diego D., Sosa D., Singular Lagrangian systems and variational constrained mechanics on Lie algebroids, Dyn. Syst. 23 (2008), 351-397, arXiv:0706.2789.

[14] Mackenzie K.C.H., General theory of Lie groupoids and Lie algebroids, London Mathematical Society Lecture Note Series, Vol. 213, Cambridge University Press, Cambridge, 2005.

[15] Marrero J.C., Martín de Diego D., Martínez E., Discrete Lagrangian and Hamiltonian mechanics on Lie groupoids, Nonlinearity 19 (2006), 1313-1348, math.DG/0506299.

[16] Marrero J.C., Martín de Diego D., Stern A., Symplectic groupoids and discrete constrained Lagrangian mechanics, Discrete Contin. Dyn. Syst. 35 (2015), 367-397, arXiv:1103.6250.

[17] Marsden J.E., Misiołek G., Ortega J.P., Perlmutter M., Ratiu T.S., Hamiltonian reduction by stages, Lecture Notes in Mathematics, Vol. 1913, Springer, Berlin, 2007.

[18] Marsden J.E., Ratiu T.S., Introduction to mechanics and symmetry. A basic exposition of classical mechanical systems, Texts in Applied Mathematics, Vol. 17, 2nd ed., Springer-Verlag, New York, 1999.

[19] Marsden J.E., Ratiu T.S., Scheurle J., Reduction theory and the Lagrange-Routh equations, J. Math. Phys. 41 (2000), 3379-3429.

[20] Marsden J.E., Scheurle J., Lagrangian reduction and the double spherical pendulum, Z. Angew. Math. Phys. 44 (1993), 17-43.

[21] Marsden J.E., Scheurle J., The reduced Euler-Lagrange equations, in Dynamics and Control of Mechanical Systems (Waterloo, ON, 1992), Fields Inst. Commun., Vol. 1, Amer. Math. Soc., Providence, RI, 1993, 139-164.

[22] Marsden J.E., West M., Discrete mechanics and variational integrators, Acta Numer. 10 (2001), 357-514.

[23] Martínez E., Lagrangian mechanics on Lie algebroids, Acta Appl. Math. 67 (2001), 295-320.

[24] Martínez E., Lie algebroids in classical mechanics and optimal control, SIGMA 3 (2007), 050, 17 pages, math-ph/0703062.

[25] Martínez E., Variational calculus on Lie algebroids, ESAIM Control Optim. Calc. Var. 14 (2008), 356-380, math-ph/0603028.

[26] Montgomery R., Isoholonomic problems and some applications, Comm. Math. Phys. 128 (1990), 565-592.

[27] Moser J., Veselov A.P., Discrete versions of some classical integrable systems and factorization of matrix polynomials, Comm. Math. Phys. 139 (1991), 217-243.

[28] Routh E.J., An elementary treatise on the dynamics of a system of rigid bodies. With numerous examples, Macmillan, Cambridge, 1860. 
[29] Stern A., Discrete Hamilton-Pontryagin mechanics and generating functions on Lie groupoids, J. Symplectic Geom. 8 (2010), 225-238, arXiv:0905.4318.

[30] Weinstein A., Lagrangian mechanics and groupoids, in Mechanics Day (Waterloo, ON, 1992), Fields Inst. Commun., Vol. 7, Amer. Math. Soc., Providence, RI, 1996, 207-231.

[31] Wong S.K., Field and particle equations for the classical Yang-Mills field and particles with isotopic spin, Il Nuovo Cimento A 65 (1970), 689-694.

[32] Yoshimura H., Marsden J.E., Dirac structures in Lagrangian mechanics. I. Implicit Lagrangian systems, J. Geom. Phys. 57 (2006), 133-156.

[33] Yoshimura H., Marsden J.E., Dirac structures in Lagrangian mechanics. II. Variational structures, J. Geom. Phys. 57 (2006), 209-250.

[34] Yoshimura H., Marsden J.E., Reduction of Dirac structures and the Hamilton-Pontryagin principle, Rep. Math. Phys. 60 (2007), 381-426. 